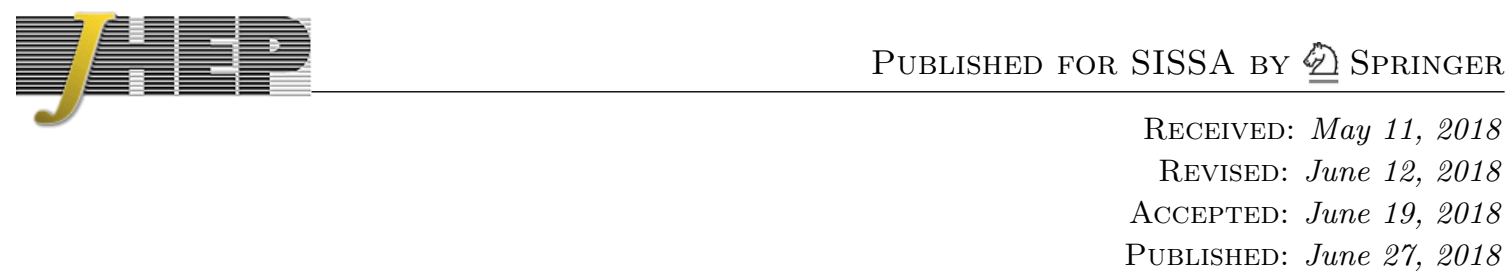

\title{
A light Higgs at the LHC and the B-anomalies
}

\author{
Da Liu, ${ }^{a}$ Jia Liu, ${ }^{b}$ Carlos E.M. Wagner ${ }^{a, b, c}$ and Xiao-Ping Wang ${ }^{a}$ \\ ${ }^{a}$ High Energy Physics Division, Argonne National Laboratory, \\ 9700 S. Cass Avenue, Argonne, IL 60439, U.S.A. \\ ${ }^{b}$ Physics Department and Enrico Fermi Institute, University of Chicago, \\ 933 East 56th Street, Chicago, IL 60637, U.S.A. \\ ${ }^{c}$ Kavli Institute for Cosmological Physics, University of Chicago, \\ 5640 South Ellis Avenue, Chicago, IL 60637, U.S.A. \\ E-mail: da.liu@anl.gov, liuj1@uchicago.edu, cwagner@anl.gov, \\ xia. wang@anl.gov
}

Abstract: After the Higgs discovery, the LHC has been looking for new resonances, decaying into pairs of Standard Model (SM) particles. Recently, the CMS experiment observed an excess in the di-photon channel, with a di-photon invariant mass of about $96 \mathrm{GeV}$. This mass range is similar to the one of an excess observed in the search for the associated production of Higgs bosons with the $Z$ neutral gauge boson at LEP, with the Higgs bosons decaying to bottom quark pairs. On the other hand, the LHCb experiment observed a discrepancy with respect to the SM expectations of the ratio of the decay of $B$-mesons to $K$-mesons and a pair of leptons, $R_{K^{(*)}}=B R\left(B \rightarrow K^{(*)} \mu^{+} \mu^{-}\right) / B R(B \rightarrow$ $\left.K^{(*)} e^{+} e^{-}\right)$. This observation provides a hint of the violation of lepton-flavor universality in the charged lepton sector and may be explained by the existence of a vector boson originating form a $\mathrm{U}(1)_{L_{\mu}-L_{\tau}}$ symmetry and heavy quarks that mix with the left-handed down quarks. Since the coupling to heavy quarks could lead to sizable Higgs di-photon rates in the gluon fusion channel, in this article we propose a common origin of these anomalies identifying a Higgs associated with the breakdown of the $\mathrm{U}(1)_{L_{\mu}-L_{\tau}}$ symmetry and at the same time responsible to the quark mixing, with the one observed at the LHC. We also discuss the constraints on the identification of the same Higgs with the one associated with the bottom quark pair excess observed at LEP.

Keywords: Beyond Standard Model, Higgs Physics, Gauge Symmetry

ARXIV EPRINT: 1805.01476 


\section{Contents}

1 Introduction $\quad 1$

2 A model with an extra U(1), light Higgs and heavy vector-like quarks 3

2.1 The Higgs sector and gauge boson sector 3

2.2 The quark sector 5

$\begin{array}{lll}2.3 & \text { Light and heavy quark interactions } & 7\end{array}$

3 Light Higgs and the CMS excess $\quad 9$

$\begin{array}{lll}3.1 & \text { Constraints from SM Higgs measurements } & 11\end{array}$

3.2 Simultaneous explanation of the CMS and LEP excesses 13

$4 \quad Z^{\prime}$ and the B-anomalies $\quad 13$

$\begin{array}{lll}4.1 & \text { Constraints on } Z^{\prime} & 14\end{array}$

5 Other constraints $\quad \mathbf{1 5}$

$\begin{array}{lll}5.1 \text { Constraints on the heavy quarks } & 15\end{array}$

$\begin{array}{lll}5.2 & \text { Constraints from flavor physics } & 17\end{array}$

6 Conclusion and summary $\quad 19$

$\begin{array}{lr}\text { A Scalar mixing parameters } & 19\end{array}$

$\begin{array}{ll}\text { B Limits from CKM matrix } & 20\end{array}$

\section{Introduction}

The discovery of a scalar resonance, with properties similar to the ones expected for the Higgs boson in the Standard Model (SM), has provided evidence for the realization of the simplest Higgs mechanism scenario of electroweak symmetry breaking. The couplings of the observed Higgs boson to the SM particles is within a few tens of percent of the ones expected within the SM. Small deviations of these coupling with respect to the SM values are still possible, and are expected in extensions of the Higgs sector that occur in most beyond the SM scenarios. For this reason, since the Higgs discovery, apart from a precise determination of the Higgs couplings, the LHC has been looking for new scalar resonances, with the di-photon channel being one of the most sensitive ones. Recently, the CMS experiment reported a $2.9 \sigma$ excess in this channel [1], with a di-photon invariant mass of about $95.3 \mathrm{GeV}$. This excess was mildly present in the $8 \mathrm{TeV}$ run [2], but became prominent only in the $13 \mathrm{TeV}$ run. While the ATLAS experiment did not observe any 
significant excess in this mass region in the $8 \mathrm{TeV}$ run [3], it has not yet reported the results of a similar search in the $13 \mathrm{TeV}$ run.

Searches for Higgs boson resonances produced in association with the $Z$ gauge boson, with Higgs bosons decaying into bottom-quark pairs, were conducted at LEP. The combination of the results of the four experiments, ALEPH, DELPHI, L3 and OPAL, led to the presence of a $2.3 \sigma$ local excess at an invariant mass of about $95-100 \mathrm{GeV}$ [4]. The agreement between the invariant mass of the excesses observed at LEP and CMS calls for a possible common origin of these two signatures [5-10].

On the other hand, the $\mathrm{LHCb}$ experiment has reported an intriguing hint of the violation of lepton-flavor universality in the decay of $B$-mesons into $K$-mesons and lepton pairs $[11,12]$, namely

$$
R_{K}=\frac{B R\left(B \rightarrow K \mu^{+} \mu^{-}\right)}{B R\left(B \rightarrow K e^{+} e^{-}\right)}=0.745_{-0.082}^{+0.097}
$$

while

$$
R_{K^{*}}=\frac{B R\left(B \rightarrow K^{*} \mu^{+} \mu^{-}\right)}{B R\left(B \rightarrow K^{*} e^{+} e^{-}\right)}=0.660_{-0.074}^{+0.113}
$$

A possible explanation of these anomalies may be provided by the introduction of a gauge boson associated with the $\mathrm{U}(1)_{L_{\mu}-L_{\tau}}$ symmetry [13-22] or other flavor symmetry [2326]. The absence of a coupling to electrons explains the deviation of the above ratios with respect to one, the value expected within the SM. In order to allow the coupling of the new gauge boson to the $B$ meson, one can introduce new vector like quarks that mix with the ordinary third and second generation quarks and that is charged under the new gauge symmetry, see the illustrative diagram in figure 1 . Such a quark mixing may be induced by the vacuum expectation value of a new Higgs boson that is neutral under the SM gauge symmetries. The coupling of this Higgs boson to the vector like quarks can induce sizable couplings to photons and gluons, which could be searched in the di-photon channel. Similarly, a mixing of such a Higgs with the SM-like Higgs boson can induce a coupling to the SM gauge bosons, while couplings to quarks are induced by Higgs and quark mixing effects. Hence, such a Higgs can also be produced in association with gauge bosons, subsequently decaying to bottom quark pairs. Therefore, a very natural question is whether such Higgs boson can be identified with the one that is observed by the CMS and LEP experiments. We analyze the signal and existing constraints in this paper and answer this interesting question in the conclusion section.

In this article, we shall describe the simplest scenario that can lead to a realization of this idea. In section 2 we shall present the model and describe the interaction of the Higgs and gauge bosons. In section 3 we shall discuss the possibility that a Higgs boson associated with the breakdown of the new $\mathrm{U}(1)_{L_{\mu}-L_{\tau}}$ symmetry leads to an explanation of the CMS excess and explain the constraints associated with a simultaneous explanation of the LEP excess. In section 4 we shall discuss the explanation of the anomalies seen in B-meson decays. In section 5 we shall concentrate on the collider and flavor physics constraints on this model. Finally, we reserve section 6 for our conclusions, and the appendices for technical details associated with the scalar mixing parameters and the obtention of the proper CKM matrix elements within this model. 


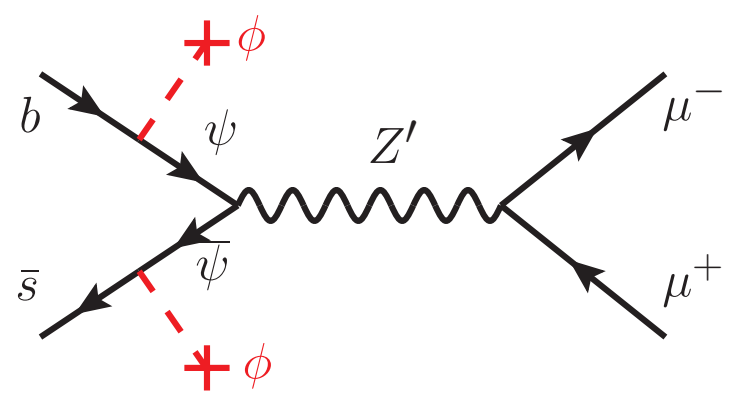

Figure 1. Feynman diagram showing how the Higgs $\phi$ induces an off-diagonal coupling of the new gauge boson $Z^{\prime}$ to the strange and bottom quarks via the mixing of the SM quarks with the heavy vector-like quark $\psi$. This process induces a contribution to the $C_{9}^{N P}$ effective operator which is responsible for the B-anomalies. A sizable coupling of the Higgs $\phi$ to di-photons and di-gluons will be induced via the coupling to the heavy quark, and hence $\phi$ could be potentially identified with the one observed by the CMS $[1,2]$ experiment in the di-photon channel.

\section{A model with an extra U(1), light Higgs and heavy vector-like quarks}

In this section, we shall introduce a gauge extension of the Standard Model (SM) to include two extra light Higgs $S, \phi$ and one extra gauge boson $Z^{\prime}$ associated with the $\mathrm{U}(1)_{L_{\mu}-L_{\tau}}$ symmetry [21, 27-50]. The gauge charge $L_{\mu}-L_{\tau}$ is not flavor universal, but diagonal. We also need one extra vector-like heavy quark $\psi_{L, R}$ charged under both SM gauge and $\mathrm{U}(1)_{L_{\mu}-L_{\tau}}$. The model is minimal to address the light Higgs and B-anomalies. The SM and new physics (NP) particles are listed in table 1 along with their gauge charges. $q_{L}^{i}\left(u_{R}^{i}, d_{R}^{i}\right)$ are the $i$ th generation left-handed (right-handed) SM quarks, where $i=1,2,3$ is the SM quark generation index. $\ell^{e, \mu, \tau}$ and $e_{R}, \mu_{R}, \tau_{R}$ are three generations of SM left-handed and right-handed leptons. $S$ and $\phi$ are NP Higgs bosons, which are singlet under the SM gauge symmetry and are responsible to give mass to $\mathrm{U}(1)_{L_{\mu}-L_{\tau}}$ gauge boson $Z^{\prime}$. The fields $\psi_{L, R}$ the chiral components of a vector-like heavy quark, which has the same SM charge as $q_{L}^{i}$ and also charged under $\mathrm{U}(1)_{L_{\mu}-L_{\tau}}$. The charge assignments of $\phi$ and $\psi$ under the $\mathrm{U}(1)_{L_{\mu}-L_{\tau}}$ symmetry need to satisfy the relation $Q_{\mu-\tau}^{\phi}= \pm Q_{\mu-\tau}^{\psi}$ to allow for the appropriate Yukawa couplings. Without loss of generality, it can be taken to be $Q_{\mu-\tau}^{\phi}=-Q_{\mu-\tau}^{\psi}=1 / 2$. The anomaly-free condition is satisfied because $\mathrm{U}(1)_{L_{\mu}-L_{\tau}}$ is vector-like in the NP particle content. It is also anomaly-free within the SM sector. The vacuum expectation value of the field $\phi$ may induce a mixing between the SM quarks and the NP heavy quarks. In the following sections, we will discuss the model in details in the Higgs, gauge boson and quark sectors.

\subsection{The Higgs sector and gauge boson sector}

In the Higgs sector of this model, we have two extra Higgs $S, \phi$. We can write down the Lagrangian for the Higgs bosons,

$$
\begin{aligned}
\mathcal{L}_{\mathrm{Higgs}}= & \left(D_{\mu} \phi\right)^{\dagger} D^{\mu} \phi+\left(D_{\mu} S\right)^{\dagger} D^{\mu} S \\
& -\mu_{\phi}^{2} \phi^{*} \phi-\lambda_{\phi}\left(\phi^{*} \phi\right)^{2}-\mu_{S}^{2} S^{*} S-\lambda_{S}\left(S^{*} S\right)^{2}-\mu^{2} H^{\dagger} H-\lambda\left(H^{\dagger} H\right)^{2} \\
& -\lambda_{\phi h}\left(\phi^{*} \phi\right)\left(H^{\dagger} H\right)-\lambda_{S h}\left(S^{*} S\right)\left(H^{\dagger} H\right)-\lambda_{S \phi}\left(S^{*} S\right)\left(\phi^{*} \phi\right) .
\end{aligned}
$$




\begin{tabular}{|c|c|c|c|c|}
\hline filed & $\mathrm{SU}(3)_{C}$ & $\mathrm{SU}(2)_{L}$ & $\mathrm{U}(1)_{Y}$ & $\mathrm{U}(1)_{L_{\mu}-L_{\tau}}$ \\
\hline$H$ & 1 & 2 & $\frac{1}{2}$ & 0 \\
\hline$q_{L}^{i}$ & 3 & 2 & $\frac{1}{6}$ & 0 \\
\hline$u_{R}^{i}$ & 3 & 1 & $\frac{2}{3}$ & 0 \\
\hline$d_{R}^{i}$ & 3 & 1 & $-\frac{1}{3}$ & 0 \\
\hline$\ell^{e}$ & 1 & 2 & $-\frac{1}{2}$ & 0 \\
\hline$\ell^{\mu}$ & 1 & 2 & $-\frac{1}{2}$ & 1 \\
\hline$\ell^{\tau}$ & 1 & 2 & $-\frac{1}{2}$ & -1 \\
\hline$e_{R}$ & 1 & 1 & -1 & 0 \\
\hline$\mu_{R}$ & 1 & 1 & -1 & 1 \\
\hline$\tau_{R}$ & 1 & 1 & -1 & -1 \\
\hline$S$ & 1 & 1 & 0 & 1 \\
\hline$\phi$ & 1 & 1 & 0 & $\frac{1}{2}$ \\
\hline$\psi_{L, R}$ & 3 & 2 & $\frac{1}{6}$ & $-\frac{1}{2}$ \\
\hline
\end{tabular}

Table 1. The particle content and their $\mathrm{SU}(3)_{C} \times \mathrm{SU}(2)_{L} \times \mathrm{U}(1)_{Y} \times \mathrm{U}(1)_{L_{\mu}-L_{\tau}}$ gauge charge. $i=1,2,3$ is the SM quark generation index. $S$ and $\phi$ are the NP Higgs, and $\psi_{L, R}$ are vector-like heavy quarks. The charge assignment $Q_{\mu-\tau}^{\phi}=-Q_{\mu-\tau}^{\psi}$ allows for the appropriate Yukawa couplings. Without loss of generality, it is taken to be $1 / 2$.

The covariant derivative $D_{\mu}=\partial_{\mu}-i g_{D} Z_{\mu}^{\prime} Q_{L_{\mu}-L_{\tau}}$, with $g_{D}$ the gauge coupling of $\mathrm{U}(1)_{L_{\mu}-L_{\tau}}$. The first line in $\mathcal{L}_{\text {Higgs }}$ are kinetic terms for $\phi$ and $S$, which give mass to $Z^{\prime}$ after symmetry breaking, and others are scalar potential terms. The couplings $\lambda_{\phi h}$, $\lambda_{S h}$ and $\lambda_{S \phi}$ define the Higgs portal terms, which induce the mixing between scalars. The scalar $\phi, S$ and SM Higgs $H$ get vevs and can be expanded near the minimum as $\phi=\frac{1}{\sqrt{2}}\left(\phi^{0}+v_{D}\right), S=\frac{1}{\sqrt{2}}\left(s^{0}+v_{S}\right)$ and $H=\left(0, \frac{1}{\sqrt{2}}\left(h^{0}+v\right)\right)$.

The gauge boson $Z^{\prime}$ has no mass mixing with the SM gauge bosons. Only the two kinetic terms in the first line of eq. (2.1) give the $Z^{\prime}$ mass, which reads

$$
m_{Z^{\prime}}=g_{D} \sqrt{\frac{1}{4} v_{D}^{2}+v_{S}^{2}} .
$$

In our analysis, we assume the observed $96 \mathrm{GeV}$ di-photon excess observed at CMS is predominantly associated with the CP-even component of $\phi^{0}$. This means $v_{D}$ is nearly the same order of the SM Higgs vev $v$. If there is no another Higgs $S$, the $Z^{\prime}$ mass is too light and severely constrained by di-jet and di-lepton constraints in section 4.1. We therefore introduce another scalar $S$ with unit charge under $\mathrm{U}(1)_{L_{\mu}-L_{\tau}}$, and that contribute to the $Z^{\prime}$ mass, as described above. Therefore, the $Z^{\prime}$ mass can be as heavy as several $\mathrm{TeV}$ with a large value of $v_{S}$ and we treat it as a free parameter thereafter. In the later analysis, we could find out $m_{Z^{\prime}} \sim 4.1 \mathrm{TeV}$, thus $m_{s^{0}}$ should be of the same order. We shall not consider the phenomenology of such a heavy Higgs, as it could easily decouple from the 
SM Higgs and $\phi$ phenomenology. In our analysis, we assume $\lambda_{S h}$ and $\lambda_{S \phi}$ small, so that complemented with the heaviness of $s^{0}$ we can neglect its mixing with the light Higgs bosons and only consider the mixing between $\mathrm{H}$ and $\phi$. On the other hand, $S$ has different $\mathrm{U}(1)_{L_{\mu}-L_{\tau}}$ charge to $\Psi_{L, R}$ and SM quarks. It will not affect the quark sector mixing and we do not need to consider it any more.

For the scalar mixing, after ignore the effect of $S$, we only consider two scalars $\phi^{0}$ and $h^{0}$ with five free parameters $\mu_{\phi}, \mu, \lambda_{\phi}, \lambda$ and $\lambda_{\phi h}$. The five parameters can be traded to five physical observables, two vevs $v_{D}$ and $v$, two masses $m_{\tilde{h}}$ and $m_{\tilde{\phi}}$, and one mixing angle $\sin \alpha . \tilde{h}$ and $\tilde{\phi}$ are the mass eigenstates and related to $\phi^{0}$ and $h^{0}$ by

$$
\left(\begin{array}{c}
h^{0} \\
\phi^{0}
\end{array}\right)=\left(\begin{array}{cc}
\cos \alpha & -\sin \alpha \\
\sin \alpha & \cos \alpha
\end{array}\right)\left(\begin{array}{c}
\tilde{h} \\
\tilde{\phi}
\end{array}\right) .
$$

The five physical observables are the more useful model parameters. The relations with the old parameters are given in appendix A.

\subsection{The quark sector}

After the scalar sector, we are going to specify the mixings between the SM quarks and heavy vector-like quarks, which are responsible to induce the flavor violating couplings needed for the explanation of the B-anomalies. The most general interactions in the quark sector are given by:

$$
\begin{aligned}
\mathcal{L}_{q}= & i \bar{q}_{L}^{i} \not D q_{L}^{i}+i \bar{u}_{R}^{i} \not D u_{R}^{i}+i \bar{d}_{R}^{i} \not D d_{R}^{i}+i \bar{\psi} \not D \psi-m_{\psi} \bar{\psi} \psi \\
& -\left(\bar{q}_{L}^{i} y_{u}^{i j} \tilde{H} u_{R}^{j}+\bar{q}_{L}^{i} y_{d}^{i j} H d_{R}^{j}+\text { H.c. }\right)-\left(\sqrt{2} \lambda_{i} \bar{q}_{L}^{i} \phi \psi_{R}+\text { H.c. }\right)
\end{aligned}
$$

where $q_{L}$ are left-handed SM quarks, $u_{R}$ and $d_{R}$ are up-type and down-type right-handed SM quarks. $\psi$ is the heavy vector-like quark, $H$ is the SM Higgs and $\phi$ is the NP Higgs. $i, j$ are the generation index, $y_{u}, y_{d}$ are the SM Yukawa couplings. $\tilde{H}$ is defined as $\tilde{H} \equiv i \sigma_{2} H^{*}$. The $\mathrm{SU}(2)_{L}$ doublet $\psi$ can be written as components $\left(\psi^{u}, \psi^{d}\right)$, which are mass degenerate. After the scalars obtained vevs, the mass and interaction terms for quarks are,

$$
\mathcal{L}_{\mathrm{Higgs}}=-\sum_{q=u, d}\left(\bar{q}_{L}^{3}, \bar{q}_{L}^{2}, \bar{\psi}_{L}^{q}\right) \cdot\left(M_{q}+\Lambda_{q}\right) \cdot\left(q_{R}^{3}, q_{R}^{2}, \psi_{R}^{q}\right)^{T}+\text { H.c. },
$$

where the sum over $q=u, d$ goes over the components of $\mathrm{SU}(2)_{L}$ doublet. Since SM quarks are not charged under $\mathrm{U}(1)_{L_{\mu}-L_{\tau}}$, their Yukawa couplings are SM-like with 9 free parameters. For simplicity, we include only the third and second generation of SM quarks together with the heavy quark $\psi$ in eq. (2.5), to illustrate the essence of NP phenomenology. To further simplify the calculation, SM Yukawa couplings are chosen to be flavor diagonal. We shall relegate to the appendix the discussion of the obtention of the CKM matrix elements, which is not relevant for our analysis. Thus, the mass matrix $M_{q}$ and interaction matrix $\Lambda_{q}$ are

$$
M_{q}=\left(\begin{array}{ccc}
m_{q^{3}} & 0 & \lambda_{3} v_{D} \\
0 & m_{q^{2}} & \lambda_{2} v_{D} \\
0 & 0 & m_{\psi}
\end{array}\right), \quad \Lambda_{q}=\left(\begin{array}{ccc}
\frac{m_{q^{3}}}{v} h^{0} & 0 & \lambda_{3} \phi^{0} \\
0 & \frac{m_{q^{2}}}{v} h^{0} & \lambda_{2} \phi^{0} \\
0 & 0 & 0
\end{array}\right)
$$


Note for the up-type quarks $u^{3}=t$ and $u^{2}=c$ and for down-quark $d^{3}=b$ and $d^{2}=s$. In the matrices $M_{q}$ and $\Lambda_{q}$, only the diagonal mass elements are modified when changing from $u$ to $d$, while the terms proportional to $\lambda_{2,3}$ stay the same. Since $\psi$ is heavy vector-like quark, the masses have the sequence $m_{\psi} \gtrsim m_{q^{3}}>m_{q^{2}}$, where as we will show $\lambda_{2} v_{D}>m_{\psi}$ is necessary to obtain a large enough mixing between heavy quarks and SM 2nd generation quarks. For the up sector, since the top quark mass is of the order of the weak scale, $m_{q^{3}}$ can be comparable with $m_{\psi}$. To avoid large mixing between the top and the 3rd generation, and hence large Yukawas to obtain the proper top mass, we further assume $\lambda_{2} \gg \lambda_{3}$ which suggests the heavy quark mostly mixes with 2nd generation left-handed SM quarks. After diagonalizing $M_{q}$, we get the mass of the quark mass-eigenstates,

$$
\begin{aligned}
& m_{\tilde{q}^{3}}^{2} \approx m_{q^{3}}^{2}-\frac{m_{q^{3}}^{2} \lambda_{3}^{2} v_{D}^{2}}{\lambda_{2}^{2} v_{D}^{2}+m_{\psi}^{2}-m_{q^{3}}^{2}}=m_{q^{3}}^{2}\left(1-\tan ^{2} \theta_{3}\right) \\
& m_{\tilde{q}^{2}}^{2} \approx m_{q^{2}}^{2} \frac{m_{\psi}^{2}}{m_{\psi}^{2}+\lambda_{2}^{2} v_{D}^{2}}=m_{q^{2}}^{2} \cos ^{2} \theta_{2} \\
& m_{\tilde{\psi}}^{2} \approx m_{\psi}^{2}+v_{D}^{2} \lambda_{2}^{2}+\frac{\lambda_{3}^{2} v_{D}^{2}\left(\lambda_{2}^{2} v_{D}^{2}+m_{\psi}^{2}\right)}{\lambda_{2}^{2} v_{D}^{2}+m_{\psi}^{2}-m_{q^{3}}^{2}}+m_{q^{2}}^{2} \frac{\lambda_{2}^{2} v_{D}^{2}}{\lambda_{2}^{2} v_{D}^{2}+m_{\psi}^{2}} \simeq m_{\psi}^{2}+\lambda_{2}^{2} v_{D}^{2}
\end{aligned}
$$

where in the last equality we only keep the leading term and we define $\tan \theta_{2} \equiv \frac{\lambda_{2} v_{D}}{m_{\psi}}$ and $\tan \theta_{3} \equiv \frac{\lambda_{3} v_{D}}{\sqrt{\lambda_{2}^{2} v_{D}^{2}+m_{\psi}^{2}-m_{q^{3}}^{2}}}$. Following the mass and coupling assumption, $\tan \theta_{3} \ll 1$. As explained above, to fit the B-meson decay anomalies, $\lambda_{2} v_{D}$ needs to be larger than $m_{\psi}$, which suggests $\sin \theta_{2}$ and $\cos \theta_{2}$ are of $\mathcal{O}(1)$. Note that the mass of $\tilde{\psi}_{u}$ and $\tilde{\psi}_{d}$ are almost degenerate, only different by small 2 nd generation quark mass $m_{q^{2}}$ and coupling $\lambda_{3}$.

The mixing matrices $U_{L, R}^{q}$ connect flavor and mass eigenstates as

$$
\left(q^{3}, q^{2}, \psi^{q}\right)_{L, R}^{T}=U_{L, R}^{q} \cdot\left(\tilde{q}^{3}, \tilde{q}^{2}, \tilde{\psi}^{q}\right)_{L, R}^{T},
$$

where the states with tilde are the mass eigenstates for the quarks. The mixing matrices at leading order are

$$
\begin{aligned}
& U_{L}^{q} \approx\left(\begin{array}{ccc}
1+\frac{1}{2} t_{\theta_{3}}^{2} & 0 & t_{\theta_{3}} \\
-s_{\theta_{2}} t_{\theta_{3}} & c_{\theta_{2}}\left(-1+\frac{m_{\tilde{q}^{2}}^{2}}{m_{\tilde{\psi}}^{2}} t_{\theta_{2}}^{2}\right) & s_{\theta_{2}}\left(1+\frac{m_{\tilde{q}^{2}}^{2}}{m_{\tilde{\psi}}^{2}}-\frac{1}{2} t_{\theta_{3}}^{2}\right) \\
-c_{\theta_{2}} t_{\theta_{3}} & s_{\theta_{2}}\left(1+\frac{m_{\tilde{q}^{2}}}{m_{\tilde{\psi}}^{2}}\right) & c_{\theta_{2}}\left(1-\frac{m_{\tilde{q}^{2}}^{2}}{m_{\tilde{\psi}}^{2}} t_{\theta_{2}}^{2}-\frac{1}{2} t_{\theta_{3}}^{2}\right)
\end{array}\right), \\
& U_{R}^{q} \approx\left(\begin{array}{ccc}
1 & \frac{m_{\tilde{q}^{2}}}{m_{\tilde{q}^{3}}} t_{\theta_{2}} t_{\theta_{3}} & \frac{m_{q^{3}}}{m_{\tilde{\psi}}} t_{\theta_{3}} \\
-\frac{m_{\tilde{q}^{2}}}{m_{\tilde{q}^{3}}} t_{\theta_{2}} t_{\theta_{3}} & 1 & \frac{m_{q^{2}}}{m_{\tilde{\psi}}} s_{\theta_{2}} \\
-\frac{m_{q^{3}}}{m_{\tilde{\psi}}} t_{\theta_{3}} & -\frac{m_{q^{2}}}{m_{\tilde{\psi}}} s_{\theta_{2}} & 1
\end{array}\right),
\end{aligned}
$$

where $s_{\theta_{2}}, c_{\theta_{2}}, t_{\theta_{2}}$ are abbreviations for $\sin \theta_{2}, \cos \theta_{2}$ and $\tan \theta_{2}$.

We see that $U_{R}^{q}$ is close to identity matrix with off-diagonal terms suppressed by small SM quark mass $m_{q^{2}, q^{3}}$ or $\tan \theta_{3}$. Because the heavy vector-like quark has the same gauge 
SM charge as the left-handed SM quarks, thus the mixing dominantly happens between the left-handed quarks. For the left-handed mixing $U_{L}^{q}$ in eq. (2.11), the leading term are the same for up-type an down-type quarks, if neglecting the SM quark masses.

The SM Cabibbo-Kobayashi-Maskawa (CKM) matrix elements should be obtained from $V_{\mathrm{CKM}} \equiv U_{L}^{u \dagger} U_{L}^{d}$. However, since we did not include off-diagonal matrix elements for the SM-quarks, all non-vanishing elements will be proportional to rations of the SMquark masses and the heavy fermion masses. In reality, the successful generation of CKM matrix needs to include the 1st generation SM quark mass and the off-diagonal terms in SM Yukawa couplings. In principle, with 9 free mass parameters in SM quark sector, there should be no problem to generate CKM matrix. We will demonstrate the generation of all the $V_{\mathrm{CKM}}$ terms in the appendix $\mathrm{B}$, and show that it does not affect the NP phenomenology we are interested here.

After the discussion of the quark sector, it is worth to mention that the charged leptons can easily get mass with diagonal SM Yukawa terms. For neutrino mass and PontecorvoMaki-Nakagawa-Sakata (PMNS) matrix under gauge interaction $\mathrm{U}(1)_{L_{\mu}-L_{\tau}}$, it is generally easy to accommodate. The reason is that $L_{\mu}-L_{\tau}$ gauge satisfies $\mu \leftrightarrow \tau$ permutation which leads to mixing angle $\theta_{23}$ close to maximal $\left(45^{\circ}\right)$ while having a vanishing mixing angle $\theta_{13}$ at leading order (see review [51]). Since neutrino mass is not relevant of the NP phenomenology we are interested in, we do not modify the lepton sector further.

\subsection{Light and heavy quark interactions}

In this subsection, the flavor eigenstate scalars and quarks are rotated into mass eigenstates. In the mass eigenstate, the Yukawa interactions for scalars and quarks are,

$$
\begin{aligned}
\mathcal{L}_{\text {Higgs }} \supset & \frac{m_{\tilde{b}}}{v}\left(\frac{v}{v_{D}} t_{\theta_{3}}^{2}\left(\frac{m_{\tilde{b}}^{2}}{m_{\tilde{\psi}}^{2}}-c_{\theta_{2}}^{2}\right) s_{\alpha}+\left(1-\frac{t_{\theta_{3}}^{2}}{2}\right) c_{\alpha}\right) \tilde{h} \tilde{\tilde{b}} \tilde{b} \\
& +\frac{m_{\tilde{b}}}{v}\left(\frac{v}{v_{D}} t_{\theta_{3}}^{2}\left(\frac{m_{\tilde{b}}^{2}}{m_{\tilde{\psi}}^{2}}-c_{\theta_{2}}^{2}\right) c_{\alpha}-\left(1-\frac{t_{\theta_{3}}^{2}}{2}\right) s_{\alpha}\right) \tilde{\phi} \tilde{\bar{b}} \tilde{b} \\
& +\frac{m_{\tilde{s}}}{v}\left(\frac{v}{v_{D}} s_{\theta_{2}}^{2} s_{\alpha}-c_{\alpha}\right) \tilde{h} \overline{\tilde{s}} \tilde{s}+\frac{m_{\tilde{s}}}{v}\left(\frac{v}{v_{D}} s_{\theta_{2}}^{2} c_{\alpha}+s_{\alpha}\right) \tilde{\phi} \overline{\tilde{s}} \tilde{s} \\
& +\frac{m_{\tilde{\psi}}}{v} \frac{v}{v_{D}} s_{\alpha}\left(s_{\theta_{2}}^{2}+\frac{t_{\theta_{3}}^{2}}{2}\left(1+c_{\theta_{2}}^{2}\right)\right) \tilde{h} \overline{\tilde{\psi}} \tilde{\psi}+\frac{m_{\tilde{\psi}}}{v} \frac{v}{v_{D}} c_{\alpha}\left(s_{\theta_{2}}^{2}+\frac{t_{\theta_{3}}^{2}}{2}\left(1+c_{\theta_{2}}^{2}\right)\right) \tilde{\phi} \overline{\tilde{\psi}} \tilde{\psi} \\
& +(b \rightarrow t, s \rightarrow c)+\ldots,
\end{aligned}
$$

where the diagonal interactions with scalars are given in eq. (2.13). The dots in the last line are for the omitted off-diagonal interactions, which dominantly opens the decay of $\tilde{\psi}$ into 2nd generation SM quarks. The decay width of process $\tilde{\psi}^{q} \rightarrow \tilde{\phi} q^{2}$ is proportional to $c_{\alpha}^{2} s_{\theta_{2}}^{2}\left(m_{\psi} / v_{D}\right)^{2}$, while the other decays $\tilde{\psi}^{q} \rightarrow \tilde{\phi} q^{3}$ or $\tilde{\psi}^{q} \rightarrow \tilde{h} q^{2}$ are suppressed by either $t_{\theta_{3}}^{2}$ or $s_{\alpha}^{2}$. The off-diagonal interactions will not contribute to scalar $\tilde{h}, \tilde{\phi}$ couplings to gluon-gluon and photon-photon, thus they are irrelevant for the NP phenomenology. 
The gauge interactions between $Z^{\prime}$ and quark mass eigenstates at leading order are given below:

$$
\begin{aligned}
\mathcal{L}_{Z^{\prime}}^{\text {quark }}= & -\frac{g_{D}}{2} t_{\theta_{3}}^{2} c_{\theta_{2}}^{2} Z_{\mu}^{\prime} \overline{\tilde{b}}_{L} \gamma^{\mu} \tilde{b}_{L}-\frac{g_{D}}{2} Z_{\mu}^{\prime} s_{\theta_{2}}^{2} \overline{\tilde{s}}_{L} \gamma^{\mu} \tilde{s}_{L} \\
& +\frac{g_{D}}{2} Z_{\mu}^{\prime}\left[\left(t_{\theta_{3}}^{2}-1\right) c_{\theta_{2}}^{2} \overline{\tilde{\psi}}_{L}^{d} \gamma^{\mu} \tilde{\psi}_{L}^{d}+\left(t_{\theta_{3}}^{2}-1\right) \overline{\tilde{\psi}}_{R}^{d} \gamma^{\mu} \tilde{\psi}_{R}^{d}\right] \\
& +\frac{g_{D}}{2} Z_{\mu}^{\prime} s_{\theta_{2}} c_{\theta_{2}} t_{\theta_{3}} \overline{\tilde{b}}_{L} \gamma^{\mu} \tilde{s}_{L}+H . c . \\
& +\frac{g_{D}}{2} Z_{\mu}^{\prime}\left[-c_{\theta_{2}}^{2} t_{\theta_{3}} \overline{\tilde{b}}_{L} \gamma^{\mu} \tilde{\psi}_{L}^{d}-\frac{m_{\tilde{b}}}{m_{\tilde{\psi}}} t_{\theta_{3}} \overline{\tilde{\sigma}}_{R} \gamma^{\mu} \tilde{\psi}_{R}^{d}\right]+H . c . \\
& +\frac{g_{D}}{2} Z_{\mu}^{\prime}\left[s_{\theta_{2}} c_{\theta_{2}}\left(\frac{t_{\theta_{3}}^{2}}{2}-1\right) \overline{\tilde{s}}_{L} \gamma^{\mu} \tilde{\psi}_{L}^{d}+\frac{m_{s}}{m_{\tilde{\psi}}} s_{\theta_{2}} \overline{\tilde{s}}_{R} \gamma^{\mu} \tilde{\psi}_{R}^{d}\right]+H . c . \\
& +(b \rightarrow t, s \rightarrow c),
\end{aligned}
$$

where the first two lines are diagonal interactions, and the lines from three to five are offdiagonal interactions with quarks. Such interactions induce $Z^{\prime}$ decay into $\overline{\tilde{\psi}}+\tilde{b}, \tilde{s}$ for our benchmark point $m_{Z^{\prime}}>m_{\tilde{\psi}}$. The interaction with up-type quarks are similar, simply by substitution $b \rightarrow t$ and $s \rightarrow c$. Note the flavor violating interaction in line three is required to solve the B-anomalies. However, it also induces $B-\bar{B}$ mixing and $b \rightarrow s \gamma$ via 1-loop, which constraints are investigated in section 5.2.

The lepton interactions of $Z^{\prime}$ are

$$
\mathcal{L}_{Z^{\prime}}^{\text {lepton }}=g_{D} Z_{\mu}^{\prime}\left[\bar{\mu} \gamma^{\mu} \mu-\bar{\tau} \gamma^{\mu} \tau+\bar{\nu}_{\mu L} \gamma^{\mu} \nu_{\mu L}-\bar{\nu}_{\tau L} \gamma^{\mu} \nu_{\tau L}\right]+H . c .,
$$

Due to the charge assignments, in the absence of mixing, the $Z^{\prime}$ coupling to leptons would be 2 times larger than the one to heavy quarks. We calculate the decay branching ratios (BR) for $Z^{\prime}$ into heavy vector-like quark $\tilde{\bar{\psi}} \tilde{\psi}$, 2nd generation SM quarks $\overline{\tilde{s}} \tilde{s}$ and $\overline{\tilde{c}} \tilde{c}$, 2nd and 3rd generation SM charged leptons $\mu^{+} \mu^{-}, \tau^{+} \tau^{-}$and neutrinos $\bar{\nu}_{\mu} \nu_{\mu}, \bar{\nu}_{\tau} \nu_{\tau}$. The width of the decay to 3 rd generation quarks are suppressed by $t_{\theta_{3}}^{4}$. The only significant flavor offdiagonal decays for $Z^{\prime}$ are to 2 nd generation quark and heavy vector-like quarks $\overline{\tilde{s}} \tilde{\psi}^{d}, \overline{\tilde{\psi}}^{d} \tilde{s}$ and $\overline{\tilde{c}} \tilde{\psi}^{u}, \overline{\tilde{\psi}}^{u} \tilde{c}$. The flavor off-diagonal decays for $Z^{\prime}$ to 3 rd generation are suppressed by $t_{\theta_{3}}^{2}$. The decay BRs for $Z^{\prime}$ are given in figure 2 .

Before closing this subsection, we calculate the leading order couplings between the SM $Z$ boson and SM quarks, which are modified due to mixing effects,

$$
\begin{aligned}
\mathcal{L}_{Z}= & \frac{g}{c_{w}}\left(-\frac{1}{2}+\frac{1}{3} s_{w}^{2}\right) Z_{\mu}\left[\overline{\tilde{b}}_{L} \gamma^{\mu} \tilde{b}_{L}+\overline{\tilde{s}}_{L} \gamma^{\mu} \tilde{s}_{L}+\overline{\tilde{\psi}}_{L}^{d} \gamma^{\mu} \tilde{\psi}_{L}^{d}\right] \\
& +g Z_{\mu}\left[\left(\frac{s_{w}^{2}}{3 c_{w}}-\frac{m_{\tilde{b}}^{2} t_{\theta_{3}}^{2}}{2 m_{\tilde{\psi}}^{2}}\right) \overline{\tilde{b}}_{R} \gamma^{\mu} \tilde{b}_{R}+\frac{s_{w}^{2}}{3 c_{w}} \overline{\tilde{s}}_{R} \gamma^{\mu} \tilde{s}_{R}+\left(-\frac{1}{2}+\frac{s_{w}^{2}}{3}+\frac{m_{\tilde{b}}^{2} t_{\theta_{3}}^{2}}{2 m_{\tilde{\psi}}^{2}}\right) \overline{\tilde{\psi}}_{R} \gamma^{\mu} \tilde{\psi}_{R}\right] \\
& +g Z_{\mu}\left[-\frac{m_{\tilde{b}}}{2 m_{\tilde{\psi}}} t_{\theta_{3}} \overline{\tilde{b}}_{R} \gamma^{\mu} \tilde{\psi}_{R}+\frac{m_{\tilde{s}}}{2 m_{\tilde{\psi}}} t_{\theta_{2}} \overline{\tilde{s}}_{R} \gamma^{\mu} \tilde{\psi}_{R}-\frac{m_{\tilde{b}} m_{\tilde{s}}}{2 m_{\tilde{\psi}}^{2}} t_{\theta_{2}} t_{\theta_{3}} \overline{\tilde{b}}_{R} \gamma^{\mu} \tilde{s}_{R}\right]+H . c . \\
& +\left(b \rightarrow t, s \rightarrow c, \frac{1}{3} \rightarrow-\frac{2}{3},-\frac{1}{2} \rightarrow \frac{1}{2}\right)
\end{aligned}
$$




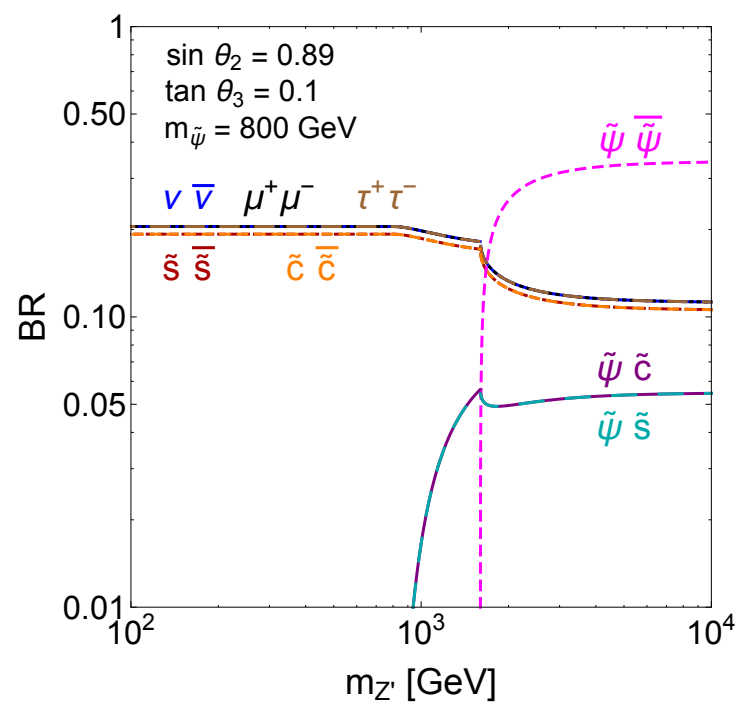

Figure 2. The $Z^{\prime}$ decay branching ratios. The label " $\bar{\nu} \nu$ " includes both $\bar{\nu}_{\mu} \nu_{\mu}$ and $\bar{\nu}_{\tau} \nu_{\tau}$. The label

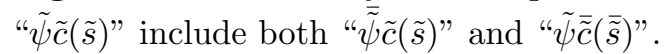

where the $Z$ coupling to left-handed quarks are exactly diagonal because the coupling of the new left-handed quarks are the same as the ones of the SM left-handed quarks. In the second line of eq. (2.16), we see the $Z$ coupling to right-handed SM quarks are approximately SM-like, apart from small corrections proportional to the quark masses. The third line contains the flavor off-diagonal interactions with $\mathrm{SM} Z$ and right-handed quarks. Although suppressed by small mixing angle $t_{\theta_{3}}$ or by quark-mass factors, it is still necessary to check flavor violating processes in the top-quark sector, e.g. $\tilde{t} \rightarrow Z \tilde{c}, Z \tilde{u}$, which are covered in section 5.2.

\section{Light Higgs and the CMS excess}

In this section, we first discuss the light Higgs $\tilde{\phi}$ with mass $96 \mathrm{GeV}$ and how it can resolve the excess in CMS data. Later, we use the $Z^{\prime}$ in the same model to fit the $R_{K}^{(*)}$ excess.

The dominantly production channels at LHC are gluon-gluon fusion (ggF), associate production with $\bar{t} t$, vector boson fusion (VBF) and associate production (VH). In the section 2.3 , the coupling between $\tilde{\phi}$ and quarks are given. For $m_{\tilde{\phi}}=96 \mathrm{GeV}$, the coupling strength $\kappa$ is calculated, which is the coupling for $\tilde{\phi}$ devided by the coupling of SM-like Higgs with the same mass.

$$
\begin{aligned}
\kappa_{\mathrm{eff}}^{\tilde{t} \tilde{\tilde{t}}(\tilde{b} \tilde{\tilde{b}}) \tilde{\phi}} & =\frac{v}{v_{D}} t_{\theta_{3}}^{2}\left(\frac{m_{\tilde{t} / \tilde{b}}^{2}-m_{\tilde{\psi}}^{2} c_{\theta_{2}}^{2}}{m_{\tilde{\psi}}^{2}}\right) c_{\alpha}-\left(1-\frac{t_{\theta_{3}}^{2}}{2}\right) s_{\alpha}, \\
\kappa_{\mathrm{eff}}^{\tilde{s} \tilde{s}(\tilde{\tilde{c}} \tilde{\tilde{c}}) \tilde{\phi}} & =\frac{v}{v_{D}} s_{\theta_{2}}^{2} c_{\alpha}+s_{\alpha}, \\
\kappa_{\mathrm{eff}}^{V V \tilde{\phi}} & =-s_{\alpha},
\end{aligned}
$$




$$
\begin{aligned}
& \kappa_{\mathrm{eff}}^{g g \tilde{\phi}}=\kappa_{\mathrm{eff}}^{\tilde{\tilde{t} \tilde{\phi}} \tilde{\phi}}+2 \frac{v}{v_{D}} c_{\alpha}\left(s_{\theta_{2}}^{2}+\frac{t_{\theta_{3}}^{2}}{2}\left(1+c_{\theta_{2}}^{2}\right)\right), \\
& \kappa_{\mathrm{eff}}^{\gamma \gamma \tilde{\phi}}=-1.31 \kappa_{\mathrm{eff}}^{V V \tilde{\phi}}+0.31 \kappa_{\mathrm{eff}}^{\tilde{\tilde{t} \tilde{\phi}} \tilde{\phi}}+0.31 \times \frac{5}{4} \frac{v}{v_{D}} c_{\alpha}\left(s_{\theta_{2}}^{2}+\frac{t_{\theta_{3}}^{2}}{2}\left(1+c_{\theta_{2}}^{2}\right)\right) \text {, }
\end{aligned}
$$

where $V=W, Z$. For $\kappa_{\text {eff }}^{g g \tilde{\phi}}$, the first term is the contribution from SM top quark, and the second term contains the contributions from heavy vector-like quarks $\tilde{\psi}^{u, d}$, therefore there is a factor of 2 . For $\kappa_{\text {eff }}^{\gamma \gamma \tilde{\phi}}$, the third term is again contribution from heavy vector-like quark, with $5 / 4$ from $\tilde{\psi}^{u, d}$ electricmagnetic charge square comparing to top quark.

The ggF, and the inclusive VBF and VH production cross-sections at $13 \mathrm{TeV}$ LHC are given below [52]

$$
\begin{aligned}
\sigma_{\mathrm{ggF}}^{\tilde{\phi}} & =76.3 \mathrm{pb} \times\left(\kappa_{\mathrm{eff}}^{g g \tilde{\phi}}\right)^{2}, \\
\sigma_{\mathrm{VBF} / \mathrm{VH}}^{\tilde{\phi}} & =10.4 \mathrm{pb} \times s_{\alpha}^{2} .
\end{aligned}
$$

The dominant decay channels of $\tilde{\phi}$ have the following decay widths [53]

$$
\begin{array}{ll}
\Gamma(\tilde{\phi} \rightarrow \overline{\tilde{b}} \tilde{b})=1.9 \mathrm{MeV} \times\left(\kappa_{\text {eff }}^{\tilde{b} \tilde{\tilde{b}} \tilde{\phi}}\right)^{2}, & \Gamma(\tilde{\phi} \rightarrow \tau \tau)=0.2 \mathrm{MeV} \times s_{\alpha}^{2}, \\
\Gamma(\tilde{\phi} \rightarrow \tilde{c} \overline{\tilde{c}})=0.09 \mathrm{MeV} \times\left(\kappa_{\mathrm{eff}}^{c \bar{c} \tilde{\phi}}\right)^{2} & \\
\Gamma(\tilde{\phi} \rightarrow g g)=0.16 \mathrm{MeV} \times\left(\kappa_{\mathrm{eff}}^{g g \tilde{\phi}}\right)^{2}, & \Gamma(\tilde{\phi} \rightarrow \gamma \gamma)=3.39 \mathrm{keV} \times\left(\kappa_{\mathrm{eff}}^{\gamma \gamma \tilde{\phi}}\right)^{2}
\end{array}
$$

The branching ratio for $\tilde{\phi}$ to $\tilde{b} \tilde{b}$ and $\gamma \gamma$ are approximately

$$
\begin{gathered}
B R(\tilde{\phi} \rightarrow \tilde{b} \overline{\tilde{b}})=\frac{1.9 \times\left(\kappa_{\text {eff }}^{\tilde{b} \tilde{b} \tilde{\phi}}\right)^{2}}{1.9 \times\left(\kappa_{\text {eff }}^{\tilde{b} \tilde{b} \tilde{\phi}}\right)^{2}+0.2 \times \sin ^{2} \alpha+0.16 \times\left(\kappa_{\text {eff }}^{g g \tilde{\phi}}\right)^{2}+0.09 \times\left(\kappa_{\text {eff }}^{\tilde{c} \tilde{\tilde{c}} \tilde{\phi}}\right)^{2}} \\
B R(\tilde{\phi} \rightarrow \gamma \gamma)=\frac{3.39 \times 10^{-3} \times\left(\kappa_{\text {eff }}^{\gamma \gamma \tilde{\phi}}\right)^{2}}{1.9 \times\left(\kappa_{\text {eff }}^{\tilde{b} \tilde{b} \tilde{\phi}}\right)^{2}+0.2 \times \sin ^{2} \alpha+0.16 \times\left(\kappa_{\text {eff }}^{g g \tilde{\phi}}\right)^{2}+0.09 \times\left(\kappa_{\text {eff }}^{\tilde{c} \tilde{\tilde{c}} \tilde{\phi}}\right)^{2}}
\end{gathered}
$$

To fit the $13 \mathrm{TeV}$ CMS di-photon excess [1] , one needs

$$
\sigma_{\mathrm{ggF}} \times \mathrm{BR}(\tilde{\phi} \rightarrow \gamma \gamma) \sim 0.085 \mathrm{pb}
$$

We show the parameter space $\left\{\sin \alpha, \sin \theta_{2}\right\}$ which fits the CMS excess in figure 3 , with $\sin \theta_{2} \approx \lambda_{2} v_{D} / m_{\tilde{\psi}}$. The cyan solid line provides $80 \%$ of CMS excess in eq. (3.10), while the two dashed line are for $60 \%$ and $100 \%$ of the excess respectively. The benchmark point is denoted by a red star in the plot, and its parameters are also listed in table 2 .

From figure 3 , the benchmark has $\sin \theta_{2}=0.89, m_{\tilde{\psi}}=\lambda_{2} v_{D} / \sin \theta_{2}=800 \mathrm{GeV}$ and $v_{D}=496 \mathrm{GeV}$. This suggests $\lambda_{2} \approx 1.4$ and the heavy quark mass dominantly comes from 


\begin{tabular}{|c|c|c|c|c|c|c|c|c|}
\hline model parameters & $m_{\tilde{\phi}}$ & $\sin \alpha$ & $\sin \theta_{2}$ & $\tan \theta_{3}$ & $m_{\tilde{\psi}}$ & $g_{D}$ & $v_{D}$ & $m_{Z^{\prime}}$ \\
\hline benchmark point & $96 \mathrm{GeV}$ & 0.1 & 0.89 & 0.1 & $800 \mathrm{GeV}$ & 1 & $492 \mathrm{GeV}$ & $4.1 \mathrm{TeV}$ \\
\hline
\end{tabular}

Table 2. The benchmark point for the signal model which fits the CMS excess and solves the B-anomalies simultaneously.

\begin{tabular}{|c|c|c|c|c|c|c|c|c|}
\hline process & $b \bar{b}$ & $\tau \bar{\tau}$ & $c \bar{c}$ & $g g$ & $\gamma \gamma$ & $W W^{*}$ & $Z Z^{*}$ & total \\
\hline $\mathrm{BR}$ & $15.9 \%$ & $1.66 \%$ & $18.23 \%$ & $63.9 \%$ & $1.8 \times 10^{-3}$ & $8.3 \times 10^{-4}$ & $1.41 \times 10^{-4}$ & 1 \\
\hline$\Gamma_{i}(\mathrm{MeV})$ & 0.019 & 0.022 & 0.002 & 0.077 & $2.18 \times 10^{-4}$ & $1 \times 10^{-4}$ & $1.7 \times 10^{-5}$ & 0.12 \\
\hline
\end{tabular}

Table 3. The decay widths and branching ratios for a $96 \mathrm{GeV} \tilde{\phi}$ and parameters set at the benchmark point values, given in table 2 .

off-diagonal term $\lambda_{2} v_{D}$. It is worth mentioning that since $\cos \theta_{2}=0.48$, the diagonal mass $m_{q^{2}}$ is larger than eigenstate mass $m_{\tilde{q}^{2}}$ due to the relationship $m_{\tilde{q}^{2}}=m_{q^{2}} \cos \theta_{2}$.

For the benchmark point, we list the $96 \mathrm{GeV}$ Higgs branching ratios in table 3 .

\subsection{Constraints from SM Higgs measurements}

In this subsection, we are going to check the limits from SM Higgs measurements. For convenience, we list the coupling strength $\kappa$ for the SM-like Higgs $\tilde{h}$ below,

$$
\begin{aligned}
& \kappa_{\mathrm{eff}}^{\tilde{t} \overline{\hat{t}}(\tilde{b} \tilde{b}) \tilde{h}}=\frac{v}{v_{D}} t_{\theta_{3}}^{2}\left(\frac{m_{\tilde{t} / \tilde{b}}^{2}-m_{\tilde{\psi}}^{2} c_{\theta_{2}}^{2}}{m_{\tilde{\psi}}^{2}}\right) s_{\alpha}+\left(1-\frac{t_{\theta_{3}}^{2}}{2}\right) c_{\alpha}, \\
& \kappa_{\mathrm{eff}}^{\tilde{s} \tilde{\tilde{s}}(\tilde{c} \tilde{\tilde{c}}) \tilde{h}}=\frac{v}{v_{D}} \tan ^{2} \theta_{2} \sin \alpha-\cos \alpha, \\
& \kappa_{\mathrm{eff}}^{V V \tilde{h}}=\cos \alpha, \\
& \kappa_{\mathrm{eff}}^{g g \tilde{h}}=\kappa_{\mathrm{eff}}^{\tilde{\tilde{t} \tilde{t} \tilde{h}}}+2 \frac{v}{v_{D}} s_{\alpha}\left(s_{\theta_{2}}^{2}+\frac{\tan ^{2} \theta_{3}}{2}\left(1+c_{\theta_{2}}^{2}\right)\right), \\
& \kappa_{\mathrm{eff}}^{\gamma \gamma \tilde{h}}=-1.31 \kappa_{\mathrm{eff}}^{V V \tilde{h}}+0.31 \kappa_{\mathrm{eff}}^{\tilde{t} \tilde{\tilde{t}} \tilde{h}}+0.31 \times \frac{5}{4} \frac{v}{v_{D}} s_{\alpha}\left(s_{\theta_{2}}^{2}+\frac{\tan ^{2} \theta_{3}}{2}\left(1+c_{\theta_{2}}^{2}\right)\right) .
\end{aligned}
$$

The most recent $13 \mathrm{TeV}\left(36 \mathrm{fb}^{-1}\right)$ constraints on SM Higgs coupling strength $\kappa$ are from CMS combined measurement [54],

$$
\begin{aligned}
& \kappa_{t}^{\mathrm{CMS} 13}=1.09_{-0.14}^{+0.14}, \quad \kappa_{W}^{\mathrm{CMS} 13}=1.12_{-0.19}^{+0.13}, \quad \kappa_{Z}^{\mathrm{CMS} 13}=0.99_{-0.11}^{+0.11}, \\
& \kappa_{g}^{\mathrm{CMS} 13}=1.14_{-0.13}^{+0.15}, \quad \kappa_{\gamma}^{\mathrm{CMS} 13}=1.07_{-0.18}^{+0.15} .
\end{aligned}
$$

This CMS analysis includes ggF, VBF, VH and ttH productions, and various SM Higgs decay modes. For ATLAS, the production modes are the same, but only $H \rightarrow \gamma \gamma$ and $H \rightarrow Z Z \rightarrow 4 \ell$ decay modes are included in the $\kappa$ analysis [55],

$$
\begin{array}{ll}
\kappa_{f}^{\text {ATLAS13 }}=0.89_{-0.15}^{+0.20}, & \kappa_{V}^{\text {ATLAS13 }}=1.03 \pm 0.06, \\
\kappa_{g}^{\text {ATLAS13 }}=1.08_{-0.10}^{+0.11}, & \kappa_{\gamma}^{\text {ATLAS13 }}=0.93_{-0.08}^{+0.09} .
\end{array}
$$



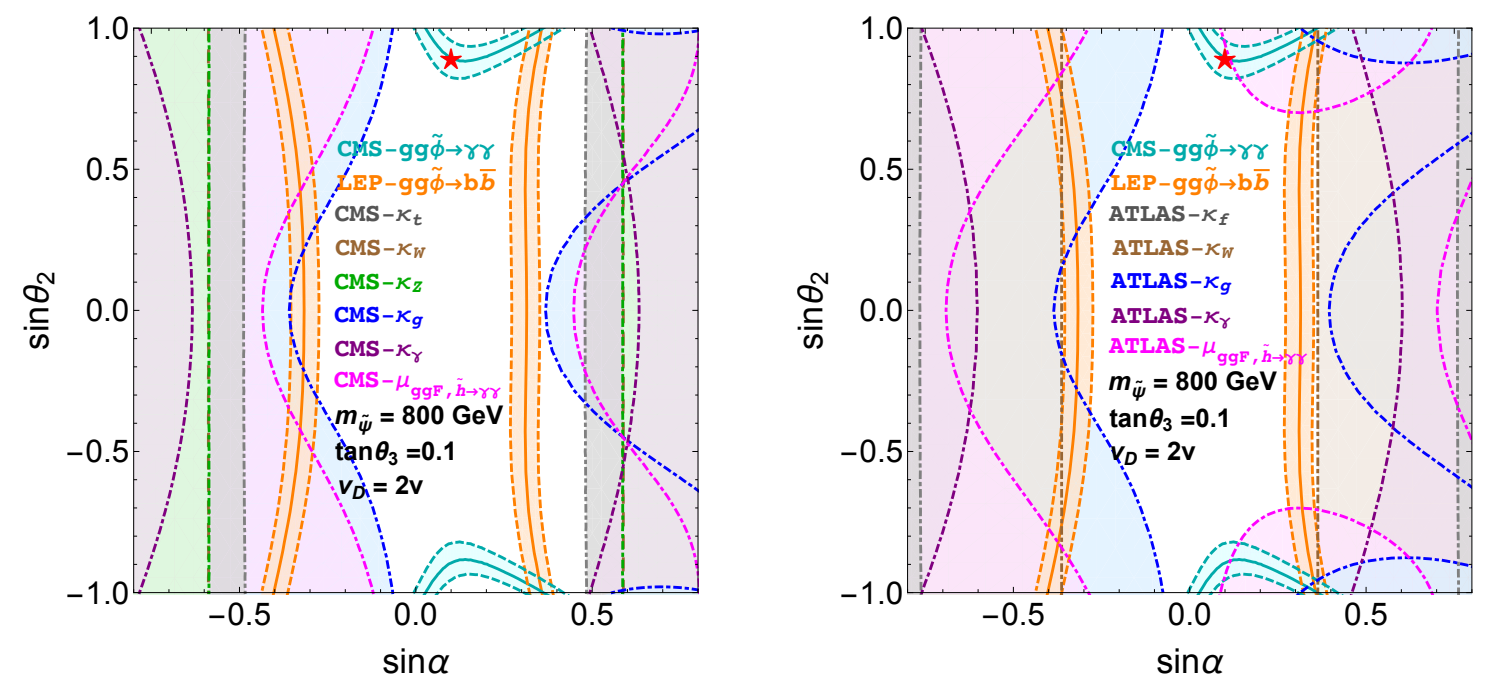

Figure 3. The $90 \%$ C.L. constraints from SM-like Higgs precision measurements and the parameter

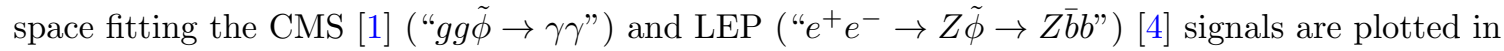
$\sin \alpha-\sin \theta_{2} 2 \mathrm{D}$ plane with cyan and orange color shading denoting the signal regions. All the other color shaded regions are excluded from various experiments. All the constraints from CMS (ATLAS) are in the left (right) panel respectively. The constraints from SM-like Higgs coupling strength, denoted as " $\kappa$ ", are from CMS [54] and ATLAS [55] measurements at $13 \mathrm{TeV}\left(36 \mathrm{fb}^{-1}\right)$. The SM Higgs di-photon decay analysis in the gluon fusion production give signal strength $\mu_{\mathrm{ggF}, h \rightarrow \gamma \gamma}$ limits for CMS [56] and ATLAS [57]. The 90\% C.L. exclusion regions are colored with dot-dashed boundaries.

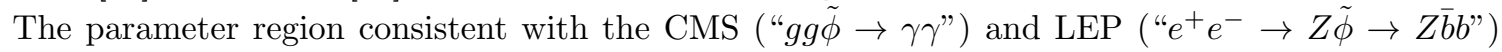
signals are the same in the left and right panels, and denoted by cyan and orange colors, respectively. The solid line shows parameters leading to an explanation of $80 \%$ of these signals, while the two dashed lines are consistent with an explanation of $60 \%$ and $100 \%$ of these signals, respectively. The red star denotes the parameters associated with the benchmark point shown in table 2 .

It is clear that $\kappa$ from CMS and ATLAS are in agreement with each other. We took the $\kappa$ measurements as constraints and plot the corresponding contours at $90 \%$ confidence level (C.L.) in figure 3, with CMS in the left panel and ATLAS in the right panel.

Since in our signal model $\tilde{\phi}$ is produced from ggF and decay in di-photon channel, it is appropriate to pay special attention in the SM Higgs di-photon channel measurement as well. The most recent $13 \mathrm{TeV}\left(36 \mathrm{fb}^{-1}\right)$ measurements for SM Higgs property in the diphoton decay channel, from CMS [56] and ATLAS [57] give the following signal strengths for different production modes and the combined result,

$$
\begin{aligned}
& \mu_{g g F, h \rightarrow \gamma \gamma}^{\mathrm{CMS} 13}=1.10_{-0.18}^{+0.20}, \\
& \mu_{t t H, h \rightarrow \gamma \gamma}^{\mathrm{CMS} 13}=2.2_{-0.8}^{+0.9}, \\
& \mu_{g g F, h \rightarrow \gamma \gamma}^{\mathrm{ATLAS} 13}=0.81_{-0.18}^{+0.19}, \\
& \mu_{t t H, h \rightarrow \gamma \gamma}^{\text {ATLAS13 }}=0.5_{-0.6}^{+0.6},
\end{aligned}
$$

$$
\begin{aligned}
\mu_{V B F, h \rightarrow \gamma \gamma}^{\mathrm{CMS} 13} & =0.8_{-0.5}^{+0.6}, \\
\mu_{\text {combined, } h \rightarrow \gamma \gamma}^{\mathrm{CMS13}} & =1.18_{-0.14}^{+0.17} \\
\mu_{V B F, h \rightarrow \gamma \gamma}^{\mathrm{ATLAS} 13} & =2.0_{-0.5}^{+0.6}, \\
\mu_{\text {combined }, h \rightarrow \gamma \gamma}^{\text {ATLAS3 }} & =0.99_{-0.14}^{+0.15} .
\end{aligned}
$$

The above measurements in the di-photon channel have improved the precision by factor of 2 compared to the ones with Run-I data [58], therefore we do not consider the Run-I 
constraints further. The most relevant and stringent constraint from the signal strengths is from $\mu_{g g F, h \rightarrow \gamma \gamma}$. The ATLAS result is barely consistent with SM at $1 \sigma$. Thus, we give 90\% C.L. $(1.64 \sigma)$ constraints for CMS and ATLAS respectively in figure 3. The excluded parameter regions are plotted as magenta color with dot-dashed boundary.

\subsection{Simultaneous explanation of the CMS and LEP excesses}

In figure 3, we show the constraints from Higgs precision measurements, as well as the region consistent with the LEP excess, shown as the orange regions in both left and right panels of figure 3. While the CMS excess can be easily explained without being in tension with any current Higgs measurements, the simultaneous explanation of the CMS di-photon and LEP $b \bar{b}$ excesses can be only obtained for values of $s_{\theta_{2}} \simeq \pm 1$ and $s_{\alpha} \simeq 0.3$. It is easy to see that, for these values of the mixing angles $\kappa_{\text {eff }}^{g g \tilde{h}} \simeq 1.2$.

The large $\kappa_{\mathrm{eff}}^{g g \tilde{h}}$ induces a sizable rate for the SM-like Higgs production in the gluon fusion channel. While such an enhancement is consistent with the current CMS measurement in the ggF channel, both in the di-photon as well as in the $Z Z$ and $W W$ final states, it is in tension with the current ATLAS measurement of the ggF production of SM-like Higgs bosons in the di-photon channel, see eq. (3.19). This tension is clearly shown in the right panel of figure 3 .

In summary, while the LEP and CMS excesses can be easily separately explained within this framework, a simultaneous explanation of the CMS and LEP excesses is in tension with the current ATLAS observations in the ggF channel. In the following, we shall concentrate on the benchmark model, which leads to an explanation of the CMS diphoton excess, and ignore the LEP $\bar{b} b$ excess. However, beyond the SM-like Higgs physics, all other phenomenological aspects of this model would be only minimally affected by a change from the benchmark point parameters to the region of parameters, where the LEP and CMS excesses are simultaneously explained.

\section{$4 \quad Z^{\prime}$ and the B-anomalies}

After discussing the possibility of $\tilde{\phi}$ fitting the CMS excess, we turn to the B-anomalies. Integrating out the heavy gauge boson $Z^{\prime}$, there is an effective flavor violating operator with down-type quarks [13],

$$
\mathcal{L}_{\text {eff }}=-\frac{g_{D}^{2}}{2 m_{Z^{\prime}}^{2}} s_{\theta_{2}} c_{\theta_{2}} t_{\theta_{3}} \overline{\tilde{b}}_{L} \gamma_{\mu} \tilde{s}_{L} \bar{\mu} \gamma^{\mu} \mu+H . c . .
$$

This can be related to the $C_{9}^{N P}$ operator considered in refs. [59-67]:

$$
\mathcal{H}_{\mathrm{eff}}^{N P}=-\mathcal{L}_{\mathrm{eff}}^{N P}=-\frac{4 G_{F}}{\sqrt{2}} \frac{\alpha_{e m}}{4 \pi}\left(V_{t b} V_{t s}^{*}\right) C_{9}^{N P} \overline{\tilde{b}}_{L} \gamma_{\mu} \tilde{s}_{L} \bar{\mu} \gamma^{\mu} \mu+H . c .,
$$

therefore, the coefficient $C_{9}^{N P}$ can be rewritten as

$$
C_{9}^{N P}=-\frac{g_{D}^{2} v^{2}}{m_{Z^{\prime}}^{2}} \frac{\pi}{\alpha_{e m}} \frac{1}{V_{t b} V_{t s}^{*}} s_{\theta_{2}} c_{\theta_{2}} t_{\theta_{3}} .
$$


Recent global fits include more data from experiments, e.g. angular observables from Belle [68], and have found that the significance of NP contributions has increased [65, 66]. If one restricts the analysis to lepton flavor universality violation process, the value $C_{9}^{N P}=-1.56$ quoted above was obtained by a fit to the data by the authors of ref. [65], with a significance of $4.1 \sigma$, while the authors of ref. [66] obtained a slightly different result, namely, $C_{9}^{N P}=-1.76$, with a significance of $3.9 \sigma$. However, extending the analysis to a more complete set of observables, namely all those included in ref. [66], a best fit value of $C_{9}^{N P}=-1.11$ is obtained, and the significance increases to $5.8 \sigma$. In our analysis, we shall consider the values obtained from the fit in ref. [65]. The alternative values of $C_{9}^{N P}$ obtained in ref. [66] do not affect our phenomenological analysis in any significant way, since they can be easily accommodated by few tens of percent changes in the mass of the gauge boson $Z^{\prime}$. After plugging in the SM parameters $\left(\alpha_{e m}=1 / 137,\left|V_{t s}\right| \sim 0.04\right)$, we obtain a requirement on NP parameters,

$$
\frac{g_{D}^{2}}{m_{Z^{\prime}}^{2}} s_{\theta_{2}} c_{\theta_{2}} t_{\theta_{3}} \sim 2.44 \times 10^{-3} \mathrm{TeV}^{-2}
$$

For the explanation of the $R_{K}$ and $R_{K}^{*}$ anomalies, we have chosen a benchmark with $t_{\theta_{3}}=0.1$ and $s_{\theta_{2}}=0.89$, which are consistent with the parameters shown by the red star in figure 3. For such a benchmark, one obtains a relationship between $g_{D}$ and $m_{Z^{\prime}}$ given by $\frac{m_{Z^{\prime}}}{g_{D}} \simeq 4.1 \mathrm{TeV}$, which is shown as the blue line in the left panel of figure 4 . In the right panel of figure 4 , we show contours of constant values of $\sin \theta_{2}$, showing the dependence on this mixing angle of the coefficient $-C_{9}^{N P}$ and the di-photon signal crosssection $\sigma_{\mathrm{ggF}} \mathrm{BR}(\tilde{\phi} \rightarrow \gamma \gamma)$ at the $13 \mathrm{TeV}$ LHC. We see that di-photon signal at CMS requires a large value of $\sin \theta_{2}$, while the explanation of the $\mathrm{B}$-anomalies requires a moderate value of $\sin \theta_{2}$ since $-C_{9}^{N P}$ is proportional to $s_{\theta_{2}} c_{\theta_{2}}$.

\subsection{Constraints on $Z^{\prime}$}

In eq. (2.14), we have already presented the interactions of $Z^{\prime}$ and in figure 2 we show the branching ratios of $Z^{\prime}$. We find out that, depending on the $Z^{\prime}$ mass, the dominant decay channel of $Z^{\prime}$ are $Z^{\prime} \rightarrow \overline{\tilde{s}} \tilde{s}(\overline{\tilde{c}} \tilde{c}), Z^{\prime} \rightarrow \overline{\tilde{\psi}}^{u} \tilde{\psi}^{u}\left(\overline{\tilde{\psi}}^{d} \tilde{\psi}^{d}\right), Z^{\prime} \rightarrow \bar{\mu} \mu(\bar{\tau} \tau)$ and $Z^{\prime} \rightarrow \nu_{\mu} \bar{\nu}_{\mu}\left(\nu_{\tau} \bar{\nu}_{\tau}\right)$.

We now consider the constraints from $Z^{\prime}$ searches at LHC. The search for new neutral gauge bosons decaying to di-lepton [69-73] and di-jet [74] put strong constraints on a possible $Z^{\prime}$. After consider the $Z^{\prime}$ production and decay branching ratios, in the left panel of figure 4 , we show the constraints in $g_{D}$ vs. $m_{Z^{\prime}} 2 \mathrm{D}$ plane with benchmark parameters $\tan \theta_{3}=0.1, \sin \theta_{2}=0.89$ and $m_{\tilde{\psi}}=800 \mathrm{GeV}$. All the colored regions are excluded. The limits from di-jet are much weaker than di-lepton, because $Z^{\prime}$ couples to SM quark only through heavy vector-like quark mixing while leptons are directly charged. Thus di-jet constraint is not shown in figure 4 . The solution to B-anomalies requires $m_{Z^{\prime}} / g_{D} \simeq 4.1 \mathrm{TeV}$. It is plotted as a solid blue line and denoted as " $R(K) / R\left(K^{*}\right)$ " in figure 4 . To evade the constraints, $Z^{\prime}$ must be heavier than about $4.1 \mathrm{TeV}$, while $g_{D}$ must be larger than about 0.8 .

For $\mathrm{U}(1)_{L_{\mu}-L_{\tau}}$ gauge boson, it is known that neutrino trident production $\nu_{\mu} N \rightarrow$ $\nu_{\mu} N \mu^{+} \mu^{-}$, where $N$ is nuclei, gives very stringent constraint [13, 75]. The recent results are from CHARM-II [76], CCFR [77] and $\mathrm{NuTeV}$ [78], which leads to constraint 

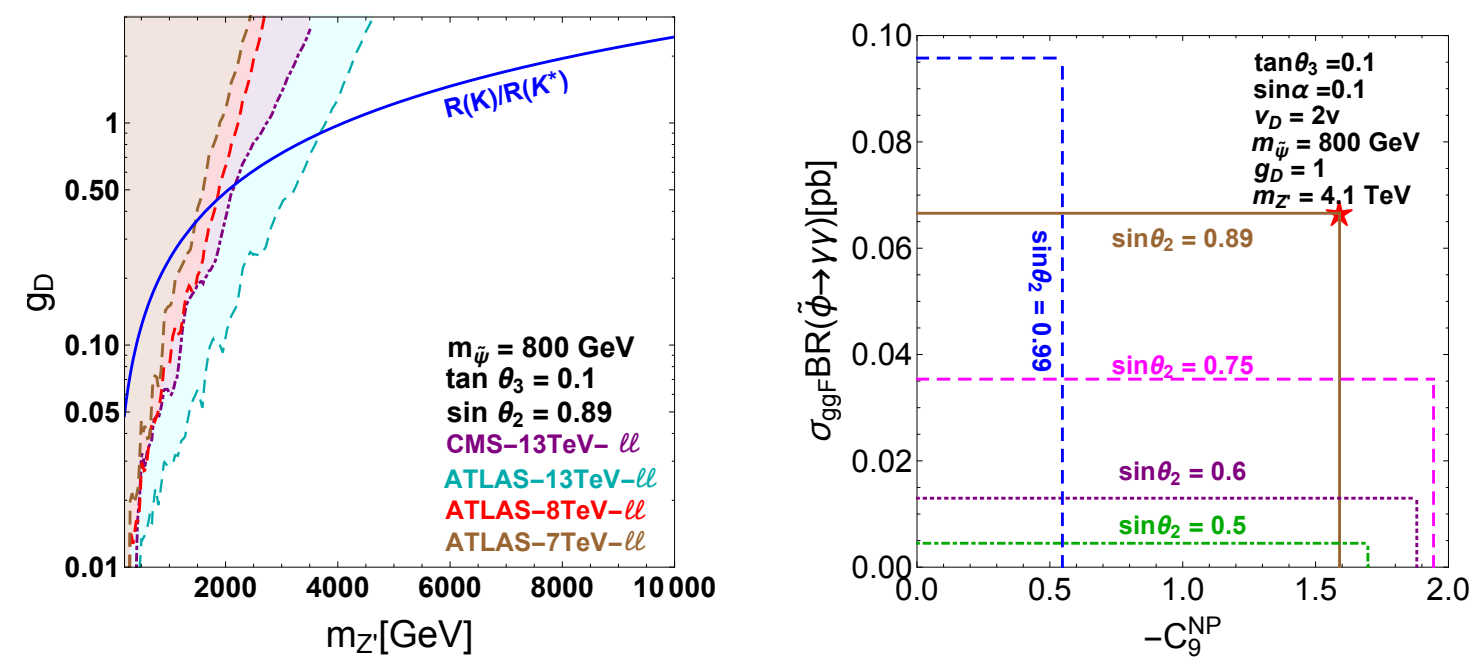

Figure 4. Left panel: the constraints on $Z^{\prime}$ in $m_{Z^{\prime}}-g_{D}$ 2D plane. The colored regions are excluded by the LHC search for heavy neutral gauge boson. The purple area is excluded by CMS $13 \mathrm{TeV}$ search on di-leptons [69]. The cyan and red areas are excluded by ATLAS $13 \mathrm{TeV}[70,71]$ and $8 \mathrm{TeV}$ di-lepton search [72]. The brown area is excluded by $7 \mathrm{TeV}$ ATLAS search on di-leptons [73]. The blue line satisfies the requirement from B-anomalies. Right panel: contours of constant values of $\sin \theta_{2}$, showing the dependence on this mixing angle of the coefficient $-C_{9}^{N P}$ and the di-photon signal cross-section $\sigma_{\mathrm{ggF}} \mathrm{BR}(\tilde{\phi} \rightarrow \gamma \gamma)$ at the $13 \mathrm{TeV}$ LHC. All the parameters except from $\sin \theta_{2}$ are taken from table 2 , and the red star denotes our benchmark point.

$m_{Z^{\prime}} / g_{D} \gtrsim 540 \mathrm{GeV}$ for $m_{Z^{\prime}} \gtrsim 10 \mathrm{GeV}$ [79]. It is easy to see that our benchmark point for model is safe from neutrino trident constraint. Observe that a simultaneous explanation of the B-anomalies and the LEP bottom forward-backward asymmetry with a single $Z^{\prime}$ is not possible, since the latter demands a light $Z^{\prime}$, with mass comparable to the $Z$ mass and significant couplings to the right-handed bottom quarks [80], what would lead to inconsistencies with the above constraints.

\section{Other constraints}

\subsection{Constraints on the heavy quarks}

The heavy vector-like quark is subject to constraints from LHC searches. Its mass is $m_{\tilde{\psi}} \simeq \sqrt{\lambda_{2}^{2} v_{D}^{2}+m_{\psi}^{2}}$, and it is about $m_{\tilde{\psi}} \simeq 800 \mathrm{GeV}$ for the benchmark point. At the LHC, such a quark would be produced by QCD processes and will predominantly decay into the $\tilde{\psi} \rightarrow \tilde{\phi} \tilde{s} / \tilde{c}$ channels, followed by $\tilde{\phi} \rightarrow \overline{\tilde{c}} \tilde{c}, \overline{\tilde{s}} \tilde{s}, \tilde{\tilde{b}} \tilde{b}$. There are other possible decay channels, e.g. $\tilde{\psi}^{q} \rightarrow \tilde{\phi} q^{3}$ and $\tilde{\psi}^{q} \rightarrow \tilde{h} q^{2}$ however suppressed by either $t_{\theta_{3}}^{2}$ or $s_{\alpha}^{2}$, and $\tilde{\psi} \rightarrow Z q$ suppressed by small quark mass or $t_{\theta_{3}}$ (see eq. (2.16)). The decay $\tilde{\psi} \rightarrow Z^{\prime} \tilde{s} / \tilde{c}$ is also possible but is kinematically forbidden by the benchmark setup $m_{Z^{\prime}}>m_{\tilde{\psi}}$. Therefore, the dominant constraints come from searches for heavy fermions which decay into three quarks. A recent relevant search is from ATLAS $13 \mathrm{TeV}\left(36 \mathrm{fb}^{-1}\right)$ looking for R-Parity violating (RPV) gluino at the LHC, which decays to three quarks [81]. It sets 95\% C.L. upper limit on 


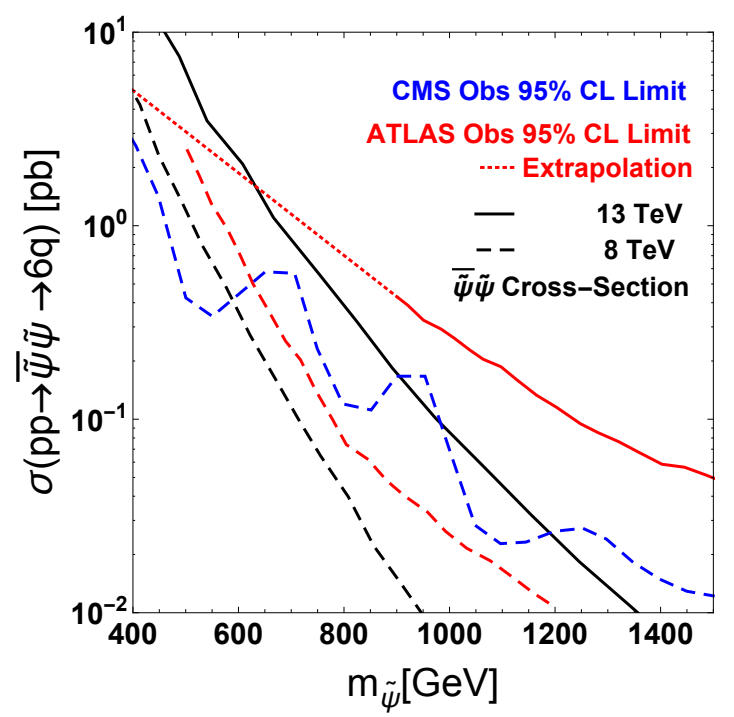

Figure 5. The 95\% C.L. constraints on the heavy vector-like quark pair production cross-section with subsequent decay into three light flavor jets. The $95 \%$ C.L. limits are from RPV gluino search at $13 \mathrm{TeV}$ ATLAS [81] and at $8 \mathrm{TeV}$ CMS [83] and ATLAS [82]. The 13 (8) TeV limits are plotted as solid (dashed) lines, and ATLAS and CMS are using red and blue color respectively. The black lines show the pair production cross-sections for $\tilde{\psi}$ at $8 \mathrm{TeV}$ and $13 \mathrm{TeV}$.

the cross section times branching ratio varies between $0.80 \mathrm{fb}$ at gluino mass $900 \mathrm{GeV}$ and $0.011 \mathrm{fb}$ at gluino mass $1800 \mathrm{GeV}$. At LHC Run-I, ATLAS [82] and CMS [83] have a similar limit for RPV gluino at $8 \mathrm{TeV}\left(20 \mathrm{fb}^{-1}\right)$, which excludes gluino mass lower than $650 \mathrm{GeV}$ if it decays into three light-flavor jets. In figure 5 , we show the pair production cross-section of $\overline{\tilde{\psi}} \tilde{\psi}$ at $13 \mathrm{TeV}$ and $8 \mathrm{TeV}[84,85]$, including both $\tilde{\psi}^{u}$ and $\tilde{\psi}^{d}$. The limits on $\sigma \times B R$ from ATLAS and CMS are plotted as red and blue lines, while $13 \mathrm{TeV}$ and $8 \mathrm{TeV}$ are using solid and dashed lines respectively. The $13 \mathrm{TeV}$ ATLAS data has stopped at $900 \mathrm{GeV}$ and we extrapolate it down to $400 \mathrm{GeV}$ with dotted line. From figure 5 , it is clear that $\tilde{\psi}$ mass larger than $600 \mathrm{GeV}$ are compatible with the $8 \mathrm{TeV}$ constraints and ATLAS $13 \mathrm{TeV}$ data requires $\tilde{\psi}$ mass larger than $650 \mathrm{GeV}$ if the extrapolation is correct. The above limits are valid for $\tilde{\psi}$ decaying to three light flavor jets. The CMS $8 \mathrm{TeV}$ search [83] has also set limit if it decays to $q q b$, and the constraint is slightly better than $q q q$ decay channel. The ATLAS $8 \mathrm{TeV}$ search [82] has looked for $b b b$ decay channel, and the constraint is roughly the same as $q q q$ channel. The heavy vector-like quark $\tilde{\psi}$ can decay to $q b b$, if $\tilde{\phi} \rightarrow \bar{b} b$. Such decay does not match to three jets invariant mass reconstruction for either $q q b$ or $b b b$, therefore more possible combinatorial errors will reduce the signal efficiency. Moreover, it will pay double suppression from branching ratio $\tilde{\phi} \rightarrow \bar{b} b$. As a result, $\tilde{\psi}$ decays into three light flavor jets are more general and useful. In summary, our benchmark model is not excluded by the three jet resonance searches at LHC. 


\subsection{Constraints from flavor physics}

We start the discussion from $b \rightarrow s \gamma$ constraints. The SM branching ratio for $b \rightarrow s \gamma$ is given by [86]:

$$
\frac{\operatorname{BR}(b \rightarrow s \gamma)}{\operatorname{BR}\left(b \rightarrow c e \bar{\nu}_{e}\right)}=\frac{6 \alpha}{\pi} \frac{\left[\eta^{\frac{16}{23}} A_{\gamma}+\frac{8}{3}\left(\eta^{\frac{14}{23}}-\eta^{\frac{16}{23}}\right) A_{g}+C\right]^{2}}{I\left(m_{c} / m_{b}\right)\left[1-\frac{2}{3 \pi} \alpha_{s}\left(m_{b}\right) f\left(m_{c} / m_{b}\right)\right]},
$$

where $\eta=\frac{\alpha_{s}\left(m_{Z}\right)}{\alpha_{s}\left(m_{b}\right)}=0.548, I\left(m_{c} / m_{b}\right)=0.58$ is the phase-space factor and $C=-0.18$ is a coefficient from the operator mixing. The Wilson coefficients $A_{\gamma}\left(m_{t}^{2} / m_{W}^{2}\right), A_{g}\left(m_{t}^{2} / m_{W}^{2}\right)$ are defined as following:

$$
\mathcal{L}_{\text {eff }}=G_{F} \frac{\alpha}{8 \pi^{3}} V_{t b} V_{t s}^{*} m_{b}\left[A_{\gamma} \bar{s}_{L} \sigma^{\mu \nu} b_{R} F_{\mu \nu}+A_{g} \bar{s}_{L} \sigma^{\mu \nu} T^{a} b_{R} G_{\mu \mu}^{a}\right]+\text { H.c. },
$$

where $F_{\mu \nu}, G_{\mu \nu}$ are the photon and gluon field strengths. The SM CKM matrix values are given by [87]:

$$
\left|V_{t b}\right|=1.009 \pm 0.031, \quad\left|V_{t s}\right|=0.04 \pm 0.0027 .
$$

For the formula for $A_{\gamma, g}$ from the SM $W$-loop, we refer to the appendix of ref. [86]. In our model, the contributions to $b \rightarrow s \gamma$ decay come from $Z^{\prime}, Z$ flavor off-diagonal couplings in eq. (2.14) and eq. (2.16). We are not going to calculate the contributions directly, but only make an estimation for the order of magnitude comparing with the contribution from $W$-loop. It is not difficult to estimate their contribution to the Wilson coefficients $A_{\gamma}, A_{g}$ :

$$
\begin{aligned}
A_{\gamma, g}^{Z} \sim & \frac{1}{V_{t b} V_{t s}^{*}} \frac{m_{W}^{2}}{m_{Z}^{2}} \frac{m_{\tilde{b}} m_{\tilde{s}}}{m_{\tilde{\psi}}^{2}}\left[A_{\gamma, g}^{\mathrm{SM}}\left(m_{\tilde{b}}^{2} / m_{Z}^{2}\right) \frac{s_{w}^{2}}{6 c_{w}}+A_{\gamma, g}^{\mathrm{SM}}\left(m_{\tilde{s}}^{2} / m_{Z}^{2}\right) \frac{s_{w}^{2}}{6 c_{w}}+\frac{1}{4} A_{\gamma, g}^{\mathrm{SM}}\left(m_{\tilde{\psi}}^{2} / m_{Z}^{2}\right)\right], \\
A_{\gamma, g}^{Z^{\prime}} \sim & \frac{1}{V_{t b} V_{t s}^{*}} \frac{g_{D}^{2} m_{W}^{2}}{4 g^{2} m_{Z^{\prime}}^{2}} t_{\theta_{3}} c_{\theta_{2}} s_{\theta_{2}} \\
& \times\left[A_{\gamma, g}^{\mathrm{SM}}\left(m_{\tilde{b}}^{2} / m_{Z^{\prime}}^{2}\right) t_{\theta_{3}}^{2} c_{\theta_{2}}^{2}+A_{\gamma, g}^{\mathrm{SM}}\left(m_{\tilde{s}}^{2} / m_{Z^{\prime}}^{2}\right) s_{\theta_{2}}^{2}+A_{\gamma, g}^{\mathrm{SM}}\left(m_{\tilde{\psi}}^{2} / m_{Z^{\prime}}^{2}\right) c_{\theta_{2}}^{2}\left(\frac{t_{\theta_{3}}^{2}}{2}-1\right)\right] .
\end{aligned}
$$

From the equation above, we can see that the contribution is either suppressed by $m_{\tilde{b}} m_{\tilde{s}} / m_{\tilde{\psi}}^{2}$ or $m_{W}^{2} / m_{Z^{\prime}}^{2}$, which leads to negligible contribution for the $b \rightarrow s \gamma$ decay. To be more explicit, we find that for $m_{Z^{\prime}} \sim 4.1 \mathrm{TeV}, g_{D} \sim 1, m_{\tilde{\psi}} \sim 800 \mathrm{GeV}$ and $t_{\theta_{3}} \ll 1$, we have:

$$
\frac{A_{\gamma}^{Z}}{A_{\gamma}^{S M}} \sim 5 \times 10^{-6}, \quad \frac{A_{g}^{Z}}{A_{g}^{S M}} \sim 4 \times 10^{-6}, \quad \frac{A_{\gamma}^{Z^{\prime}}}{A_{\gamma}^{S M}} \sim 3 \times 10^{-4} t_{\theta_{3}} c_{\theta_{2}}^{3} s_{\theta_{2}}, \quad \frac{A_{g}^{Z^{\prime}}}{A_{g}^{S M}} \sim 4 \times 10^{-4} t_{\theta_{3}} c_{\theta_{2}}^{3} s_{\theta_{2}},
$$

which are very small and do not induce any relevant modification to the $b \rightarrow s \gamma$ decay rate. Note that the operator which explains the lepton-flavor university violation in eq. (4.2) will not contribute to $B_{s} \rightarrow \mu^{+} \mu^{-}$decay, as our $Z^{\prime}$ couplings to muon pair is vector-like [88].

Apart from the operator $\overline{\tilde{b}}_{L} \gamma^{\mu} \tilde{s}_{L} \bar{\mu} \gamma_{\mu} \mu$, there are also $\Delta F=2$ flavor changing operators generated:

$$
\mathcal{L}_{\text {eff }}=-\frac{g_{D}^{2}}{4 m_{Z^{\prime}}^{2}} s_{\theta_{2}}^{2} c_{\theta_{2}}^{2} t_{\theta_{3}}^{2}\left(\overline{\tilde{b}}_{L} \gamma^{\mu} \tilde{s}_{L}\right)^{2}-\frac{g^{2}}{4 m_{Z}^{2}} \frac{m_{\tilde{b}}^{2} m_{\tilde{s}}^{2}}{m_{\tilde{\psi}}^{4}} t_{\theta_{2}}^{2} t_{\theta_{3}}^{2}\left(\overline{\tilde{b}}_{R} \gamma^{\mu} \tilde{s}_{R}\right)^{2}+H . c . .
$$


Both operators will contribute to $\bar{B}_{s}-B_{s}$ mixing, but we expect that the left-handed one dominates, as the right-handed one is suppressed by the masses of bottom and strange quarks, i.e.:

$$
\frac{m_{\tilde{b}}^{2} m_{\tilde{s}}^{2}}{m_{\tilde{\psi}}^{4}} \sim \frac{2 \times 10^{-13}}{\left(m_{\tilde{\psi}} / \mathrm{TeV}\right)^{4}}
$$

The bound on the Wilson coefficient of the first operator is given by (see table 1.1 of ref. [89] and $[90])$ :

$$
\frac{g_{D}^{2}}{4 m_{Z^{\prime}}^{2}} s_{\theta_{2}}^{2} c_{\theta_{2}}^{2} t_{\theta_{3}}^{2}<5.0 \times 10^{-5} \mathrm{TeV}^{-2}
$$

Combined with eq. (4.4), we can derive the bounds on the combination of the mixing angles or the factor $g_{D}^{2} / m_{Z^{\prime}}^{2}$ :

$$
s_{\theta_{2}} c_{\theta_{2}} t_{\theta_{3}}<0.082 \quad \Rightarrow \quad m_{Z^{\prime}}<g_{D} \times 5.8 \mathrm{TeV}
$$

which is satisfied by our benchmark point $\left(s_{\theta_{2}} c_{\theta_{2}} t_{\theta_{3}} \simeq 0.041, m_{Z^{\prime}} / g_{D} \simeq 4.1 \mathrm{TeV}\right)$.

Next, we consider the searches for $t Z$ flavor changing neutral current in the top quark decays at LHC. The relevant interactions are

$$
\mathcal{L}_{t Z}=g \frac{m_{\tilde{t}} m_{\tilde{c}}}{2 m_{\tilde{\psi}}^{2}} t_{\theta_{2}} t_{\theta_{3}} Z_{\mu} \overline{\tilde{t}}_{R} \gamma^{\mu} \tilde{c}_{R}+\text { H.c. }
$$

which will contribute to the decay channel $\tilde{t} \rightarrow Z \tilde{c}$ with branching ratio estimated as:

$$
\operatorname{BR}(\tilde{t} \rightarrow Z \tilde{c}) \sim \frac{m_{\tilde{t}}^{2} m_{\tilde{c}}^{2}}{2 m_{\tilde{\psi}}^{4}} t_{\theta_{2}}^{2} t_{\theta_{3}}^{2}
$$

The most stringent bound on such branching ratio comes from ATLAS search at $13 \mathrm{TeV}$ $\left(36 \mathrm{fb}^{-1}\right)$ [91]. The results read

$$
\operatorname{BR}(\tilde{t} \rightarrow Z \tilde{c})<2.3 \times 10^{-4}
$$

which sets a lower bound on our parameters,

$$
\frac{m_{\tilde{\psi}}}{\sqrt{t_{\theta_{2}} t_{\theta_{3}}}}>102 \mathrm{GeV}
$$

It is easy to see that such bound is satisfied in the region of parameters consistent with our benchmark point.

Finally, we discuss the constraints from flavor physics in the lepton sector. The gauge boson $Z^{\prime}$ under $\mathrm{U}(1)_{L_{\mu}-L_{\tau}}$ can contribute to tau lepton decay $\tau \rightarrow \mu \nu_{\tau} \bar{\nu}_{\mu}$ via 1-loop box diagram [13]. It excludes $Z^{\prime}$ mass lower than $650 \mathrm{GeV}$ for $g_{D}=1$, and the benchmark point with a $4 \mathrm{TeV} Z^{\prime}$ is quite safe with this limit. 


\section{Conclusion and summary}

The recently observed anomalies in the decay of $B$-mesons into $K$-mesons and pair of leptons could be a possible hint of the violation of lepton-universality, one of the pillars of the SM phenomenology. In this work, we have provided a possible realization of the idea of explaining these anomalies by the presence of a new gauge boson $Z^{\prime}$ of an $\mathrm{U}(1)_{L_{\mu}-L_{\tau}}$ symmetry, that couple to leptons at tree-level, and to quarks via the mixing with vector-like quarks charged under the new gauge symmetry. The mixing of SM quarks with vector-like quarks needs to be induced by the presence of a new Higgs boson, charged under the new $\mathrm{U}(1)_{L_{\mu}-L_{\tau}}$ symmetry.

In this work, we have discussed the possibility that such Higgs boson could be associated with the recent excess in the di-photon channel observed at the CMS experiment at the $13 \mathrm{TeV}$ LHC run. We have shown that this is possible and provided the constraints on the quark-mixing parameters that could lead to such a possibility. An additional, naturally heavy, Higgs boson, contributing to the breakdown of the $\mathrm{U}(1)_{L_{\mu}-L_{\tau}}$ symmetry, but not to the quark mixing, is necessary in order to give the $Z^{\prime}$ gauge boson a sufficiently high mass to avoid the LHC constraints. A simultaneous explanation of these observables with the Higgs boson excess observed at LEP may be achieved in a way that is consistent with most observations, although it is in tension with the rate of the SM-like Higgs boson decaying to di-photons in the gluon fusion production channel measured by the ATLAS experiment in the $13 \mathrm{TeV}$ LHC run.

\section{Acknowledgments}

Work at University of Chicago is supported in part by U.S. Department of Energy grant number DE-FG02-13ER41958. Work at ANL is supported in part by the U.S. Department of Energy under Contract No. DE-AC02-06CH11357. The work of DL was partially performed at the Aspen Center for Physics, which is supported by National Science Foundation grant PHY-1607611. We would like to thank Zhen Liu, Emmanuel Stamou, LianTao Wang for useful discussions and comments. JL acknowledges support by an Oehme Fellowship.

\section{A Scalar mixing parameters}

In this section, we give the relation between the five free parameters $\mu_{\phi}, \mu, \lambda_{\phi}, \lambda, \lambda_{\phi h}$ and the five physical observables $v_{D}, v, m_{\tilde{h}}, m_{\tilde{\phi}}, \sin \theta$. The relation is given below,

$$
\begin{array}{rlrl}
v_{D} & =\left(-\frac{\mu_{\phi}^{2}}{\lambda_{\phi}}\right)^{\frac{1}{2}}, & v & =\left(-\frac{\mu^{2}}{\lambda}\right)^{\frac{1}{2}}, \\
m_{h}=\sqrt{2} \mu=-\sqrt{2} \lambda v, & m_{\phi}=\sqrt{2} \mu_{\phi}=-\sqrt{2} \lambda_{\phi} v_{D}, \\
m_{\tilde{h}}^{2}=\frac{1}{2}\left(m_{\phi}^{2}+m_{h}^{2}+\sqrt{\left(m_{h}^{2}-m_{\phi}^{2}\right)^{2}+4 \lambda_{\phi h}^{2} v^{2} v_{D}^{2}}\right), &
\end{array}
$$




$$
\begin{aligned}
m_{\tilde{\phi}}^{2} & =\frac{1}{2}\left(m_{\phi}^{2}+m_{h}^{2}-\sqrt{\left(m_{h}^{2}-m_{\phi}^{2}\right)^{2}+4 \lambda_{\phi h}^{2} v^{2} v_{D}^{2}}\right), \\
\tan 2 \alpha & =\frac{2 \lambda_{\phi h} v v_{D}}{m_{h}^{2}-m_{\phi}^{2}} .
\end{aligned}
$$

\section{B Limits from CKM matrix}

The most general interactions for $\mathrm{U}(1)_{\mu-\tau}$ within the quark sector are given by:

$$
\begin{aligned}
\mathcal{L}_{q}= & i \bar{q}_{L}^{i} \not D q_{L}^{i}+i \bar{u}_{R}^{i} \not D u_{R}^{i}+i \bar{d}_{R}^{i} \not D d_{R}^{i}+i \bar{\psi} \not D \psi-m_{\psi} \bar{\psi} \psi-\left(\bar{q}_{L}^{i} y_{u}^{i j} \tilde{H} u_{R}^{j}+\bar{q}_{L}^{i} y_{d}^{i j} H d_{R}^{j}+\text { H.c. }\right) \\
& +\lambda_{1} \overline{q_{L}^{1}} \phi^{\star} \psi_{R}+\lambda_{2} \bar{q}_{L}^{2} \phi^{\star} \psi_{R}+\lambda_{3} \bar{q}_{L}^{3} \phi \psi_{R}+H . c .
\end{aligned}
$$

The mass matrix to generate CKM matrix in the quark sector are written in the basis of $\left(q_{3}, q_{2}, q_{1}, \psi\right)$. For up-type and down-type quarks, the mass matrices are

$$
M_{u}=\left(\begin{array}{cccc}
m_{t} & 0 & 0 & \lambda_{3} v_{D} \\
m_{23}^{u} & m_{c} & 0 & \lambda_{2} v_{D} \\
0 & 0 & m_{u} & \lambda_{1} v_{D} \\
0 & 0 & 0 & m_{\psi}
\end{array}\right), \quad M_{d}=\left(\begin{array}{cccc}
m_{b} & 0 & 0 & \lambda_{3} v_{D} \\
0 & m_{s} & 0 & \lambda_{2} v_{D} \\
m_{13}^{d} & m_{12}^{d} & m_{d} & \lambda_{1} v_{D} \\
0 & 0 & 0 & m_{\psi}
\end{array}\right) .
$$

In principle, all the matrix elements except the fourth line can be non-zero. Here we consider the simple matrices in eq. (B.2) to generate correct CKM matrix, with only three nonzero parameters in the off-diagonal terms for SM quark mass. To avoid modifying our existing results, we can set $\lambda_{1}=0$. Our notation is $M_{\text {diagonal }}=U_{L}^{\dagger} M U_{R}$, thus $q_{R}^{\text {flavor }}=U_{R} q_{R}^{\text {mass }}$ and $q_{L}^{\text {flavor }}=U_{L} q_{L}^{\text {mass }}$. Therefore, we have $\bar{u}_{L}^{\text {flavor }} \gamma^{\mu} W_{\mu}^{+} d_{L}^{\text {flavor }}=\bar{u}_{L}^{\text {mass }} \gamma^{\mu} W_{\mu}^{+} V_{\mathrm{CKM}} d_{L}^{\text {mass }}$, which results in the CKM matrix $V_{\mathrm{CKM}}=U_{L}^{u \dagger} U_{L}^{d}$. The mass of quarks are slightly changed by the small non-zero off-diagonal terms $m_{23}^{u}, m_{13}^{d}$ and $m_{12}^{d}$. The SM quark masses at leading order are

$$
\begin{array}{ll}
m_{\tilde{t}}^{2}=m_{t}^{2}-\frac{v_{D}^{2}\left(m_{23}^{u} \lambda_{2}+m_{t} \lambda_{3}\right)^{2}}{v_{D}^{2} \lambda_{2}^{2}+m_{\psi}^{2}-m_{t}^{2}}+\left(m_{23}^{u}\right)^{2}, & m_{\tilde{c}}^{2}=m_{c}^{2} \cos ^{2} \theta_{2}, \quad m_{\tilde{u}}=m_{u} \\
m_{\tilde{b}}^{2}=m_{b}^{2}\left(1-\frac{\lambda_{3}^{2}}{\lambda_{2}^{2}}\right)+\left(m_{13}^{d}\right)^{2}, & m_{\tilde{s}}^{2}=\cot ^{2} \theta_{2} \frac{m_{d}^{2}}{\left(m_{12}^{d}\right)^{2}+m_{d}^{2}} m_{s}^{2}, \\
m_{\tilde{d}}^{2}=m_{d}^{2}+\left(m_{12}^{d}\right)^{2}\left(1+\cot ^{2} \theta_{2} \frac{m_{s}^{2}}{m_{d}^{2}+\left(m_{12}^{d}\right)^{2}}\right) . &
\end{array}
$$

The heavy vector-like quark mass are the same as in eq. (2.9). The expressions for offdiagonal CKM matrix at leading order are given by,

$$
\begin{aligned}
V_{u b} & =\frac{m_{13}^{d}}{m_{b}}, \\
V_{u s} & =\frac{m_{12}^{d} m_{s} \cot \theta_{2}}{m_{12}^{d, 2}+m_{d}^{2}},
\end{aligned}
$$




$$
\begin{aligned}
V_{c d} & =-\cot \theta_{2} \frac{m_{12}^{d} m_{s}}{\left(m_{12}^{d}\right)^{2}+m_{d}^{2}}-\cot \theta_{2} \frac{m_{13}^{d} m_{23}^{u}}{m_{b} m_{t}} \simeq-V_{u s}, \\
V_{c b} & =\cot \theta_{2} \frac{m_{23}^{u}}{m_{t}} \\
V_{t s} & =-\cot \theta_{2} \frac{m_{23}^{u}}{m_{t}}-\cot \theta_{2} \frac{m_{13}^{d}}{m_{b}} \frac{m_{12}^{d} m_{s}}{\left(m_{12}^{d}\right)^{2}+m_{d}^{2}} \simeq-V_{c b}, \\
V_{t d} & =\frac{m_{23}^{u} \cot \theta_{2}}{m_{t}} \frac{m_{12}^{d} m_{s} \cot \theta_{2}}{m_{12}^{d, 2}+m_{d}^{2}}-\frac{m_{13}^{d, \star}}{m_{b}} \simeq V_{c b} \times V_{u s}-V_{u b}^{\star} .
\end{aligned}
$$

We require $\left|V_{u s}\right| \sim\left|V_{c d}\right| \simeq 0.225,\left|V_{c b}\right| \sim\left|V_{t s}\right| \simeq 0.043,\left|V_{u b}\right| \sim 0.004$ to satisfy the CKM matrix from PDG [87]. To realize $\left|V_{t d}\right| \sim 0.008$, we need $m_{13}^{d}$ to be complex number. For our benchmark point $\cot \theta_{2} \sim 0.512$, the non-zero off-diagonal SM quark mass required by CKM matrix are

$$
m_{12}^{d} \simeq 0.01 m_{\tilde{d}}, \quad\left|m_{13}^{d}\right| \simeq 0.004 m_{\tilde{b}}, \quad m_{23}^{u} \simeq 0.084 m_{\tilde{t}} .
$$

It is clear that they are sufficiently small comparing with the diagonal masses. Thus we successfully demonstrate that the generation of CKM matrix does not affect the new physics phenomenology we have discussed previously.

Open Access. This article is distributed under the terms of the Creative Commons Attribution License (CC-BY 4.0), which permits any use, distribution and reproduction in any medium, provided the original author(s) and source are credited.

\section{References}

[1] CMS collaboration, Search for new resonances in the diphoton final state in the mass range between 70 and $110 \mathrm{GeV}$ in pp collisions at $\sqrt{s}=8$ and $13 \mathrm{TeV}$, CMS-PAS-HIG-17-013, CERN, Geneva, Switzerland, (2017).

[2] CMS collaboration, Search for new resonances in the diphoton final state in the mass range between 80 and $110 \mathrm{GeV}$ in pp collisions at $\sqrt{s}=8 \mathrm{TeV}$, CMS-PAS-HIG-14-037, CERN, Geneva, Switzerland, (2014).

[3] ATLAS collaboration, Search for scalar diphoton resonances in the mass range 65-600 GeV with the ATLAS detector in pp collision data at $\sqrt{s}=8 \mathrm{TeV}$, Phys. Rev. Lett. 113 (2014) 171801 [arXiv: 1407.6583] [INSPIRE].

[4] OPAL, DelPhi, LeP Working Group for Higgs boson searches, ALEPH and L3 collaborations, R. Barate et al., Search for the Standard Model Higgs boson at LEP, Phys. Lett. B 565 (2003) 61 [hep-ex/0306033] [INSPIRE].

[5] J. Cao, X. Guo, Y. He, P. Wu and Y. Zhang, Diphoton signal of the light Higgs boson in natural NMSSM, Phys. Rev. D 95 (2017) 116001 [arXiv:1612.08522] [INSPIRE].

[6] A. Mariotti, D. Redigolo, F. Sala and K. Tobioka, New LHC bound on low-mass diphoton resonances, arXiv:1710.01743 [INSPIRE].

[7] A. Crivellin, J. Heeck and D. Müller, Large $h \rightarrow$ bs in generic two-Higgs-doublet models, Phys. Rev. D 97 (2018) 035008 [arXiv:1710.04663] [INSPIRE]. 
[8] P.J. Fox and N. Weiner, Light signals from a lighter Higgs, arXiv:1710.07649 [INSPIRE].

[9] U. Haisch and A. Malinauskas, Let there be light from a second light Higgs doublet, JHEP 03 (2018) 135 [arXiv:1712.06599] [INSPIRE].

[10] R. Vega, R. Vega-Morales and K. Xie, Light (and darkness) from a light hidden Higgs, arXiv: 1805.01970 [INSPIRE].

[11] LHCb collaboration, Test of lepton universality using $B^{+} \rightarrow K^{+} \ell^{+} \ell^{-}$decays, Phys. Rev. Lett. 113 (2014) 151601 [arXiv: 1406.6482] [INSPIRE].

[12] LHCb collaboration, Test of lepton universality with $B^{0} \rightarrow K^{* 0} \ell^{+} \ell^{-}$decays, JHEP 08 (2017) 055 [arXiv : 1705.05802] [INSPIRE].

[13] W. Altmannshofer, S. Gori, M. Pospelov and I. Yavin, Quark flavor transitions in $L_{\mu}-L_{\tau}$ models, Phys. Rev. D 89 (2014) 095033 [arXiv:1403.1269] [InSPIRE].

[14] A. Crivellin, G. D'Ambrosio and J. Heeck, Explaining $h \rightarrow \mu^{ \pm} \tau^{\mp}, B \rightarrow K^{*} \mu^{+} \mu^{-}$and $B \rightarrow K \mu^{+} \mu^{-} / B \rightarrow K e^{+} e^{-}$in a two-Higgs-doublet model with gauged $L_{\mu}-L_{\tau}$, Phys. Rev. Lett. 114 (2015) 151801 [arXiv: 1501.00993] [INSPIRE].

[15] W. Altmannshofer and I. Yavin, Predictions for lepton flavor universality violation in rare $B$ decays in models with gauged $L_{\mu}-L_{\tau}$, Phys. Rev. D 92 (2015) 075022 [arXiv:1508.07009] [INSPIRE].

[16] W. Altmannshofer, M. Carena and A. Crivellin, $L_{\mu}-L_{\tau}$ theory of Higgs flavor violation and $(g-2)_{\mu}$, Phys. Rev. D 94 (2016) 095026 [arXiv: 1604.08221] [InSPIRE].

[17] W. Altmannshofer, S. Gori, S. Profumo and F.S. Queiroz, Explaining dark matter and B decay anomalies with an $L_{\mu}-L_{\tau}$ model, JHEP 12 (2016) 106 [arXiv: 1609.04026] [INSPIRE].

[18] R. Alonso, P. Cox, C. Han and T.T. Yanagida, Flavoured B - L local symmetry and anomalous rare $B$ decays, Phys. Lett. B 774 (2017) 643 [arXiv:1705.03858] [INSPIRE].

[19] C. Bonilla, T. Modak, R. Srivastava and J.W.F. Valle, $\mathrm{U}(1)_{B_{3}-3 L_{\mu}}$ gauge symmetry as the simplest description of $b \rightarrow s$ anomalies, arXiv:1705.00915 [INSPIRE].

[20] T. Nomura and H. Okada, Zee-Babu type model with $\mathrm{U}(1)_{L_{\mu}-L_{\tau}}$ gauge symmetry, Phys. Rev. D 97 (2018) 095023 [arXiv: 1803.04795] [INSPIRE].

[21] C.-H. Chen and T. Nomura, Penguin $b \rightarrow s \ell^{\prime+} \ell^{\prime-}$ and B-meson anomalies in a gauged $L_{\mu}-L_{\tau}$, Phys. Lett. B 777 (2018) 420 [arXiv:1707.03249] [INSPIRE].

[22] P. Ko, T. Nomura and H. Okada, Explaining $B \rightarrow K^{(*)} \ell^{+} \ell^{-}$anomaly by radiatively induced coupling in $\mathrm{U}(1)_{\mu-\tau}$ gauge symmetry, Phys. Rev. D 95 (2017) 111701 [arXiv:1702.02699] [INSPIRE].

[23] A. Crivellin, G. D'Ambrosio and J. Heeck, Addressing the LHC flavor anomalies with horizontal gauge symmetries, Phys. Rev. D 91 (2015) 075006 [arXiv:1503.03477] [INSPIRE].

[24] A. Celis, J. Fuentes-Martin, M. Jung and H. Serodio, Family nonuniversal Z' models with protected flavor-changing interactions, Phys. Rev. D 92 (2015) 015007 [arXiv:1505.03079] [INSPIRE].

[25] A. Falkowski, M. Nardecchia and R. Ziegler, Lepton flavor non-universality in B-meson decays from a $\mathrm{U}(2)$ flavor model, JHEP 11 (2015) 173 [arXiv:1509.01249] [INSPIRE].

[26] A.K. Alok, B. Bhattacharya, A. Datta, D. Kumar, J. Kumar and D. London, New physics in $b \rightarrow s \mu^{+} \mu^{-}$after the measurement of $R_{K^{*}}$, Phys. Rev. D 96 (2017) 095009 [arXiv: 1704.07397] [INSPIRE]. 
[27] X.-G. He, G.C. Joshi, H. Lew and R.R. Volkas, Simplest Z' model, Phys. Rev. D 44 (1991) 2118 [INSPIRE].

[28] S. Baek, N.G. Deshpande, X.G. He and P. Ko, Muon anomalous $g-2$ and gauged $L_{\mu}-L_{\tau}$ models, Phys. Rev. D 64 (2001) 055006 [hep-ph/0104141] [INSPIRE].

[29] R. Foot, X.G. He, H. Lew and R.R. Volkas, Model for a light $Z^{\prime}$ boson, Phys. Rev. D 50 (1994) 4571 [hep-ph/9401250] [INSPIRE].

[30] E. Salvioni, A. Strumia, G. Villadoro and F. Zwirner, Non-universal minimal $Z^{\prime}$ models: present bounds and early LHC reach, JHEP 03 (2010) 010 [arXiv:0911.1450] [INSPIRE].

[31] J. Heeck and W. Rodejohann, Gauged $L_{\mu}-L_{\tau}$ symmetry at the electroweak scale, Phys. Rev. D 84 (2011) 075007 [arXiv:1107.5238] [INSPIRE].

[32] W.-Z. Feng, P. Nath and G. Peim, Cosmic coincidence and asymmetric dark matter in a Stueckelberg extension, Phys. Rev. D 85 (2012) 115016 [arXiv: 1204.5752] [INSPIRE].

[33] K. Harigaya, T. Igari, M.M. Nojiri, M. Takeuchi and K. Tobe, Muon $g-2$ and LHC phenomenology in the $L_{\mu}-L_{\tau}$ gauge symmetric model, JHEP 03 (2014) 105 [arXiv: 1311.0870] [INSPIRE].

[34] E. Ma, D.P. Roy and S. Roy, Gauged $L_{\mu}-L_{\tau}$ with large muon anomalous magnetic moment and the bimaximal mixing of neutrinos, Phys. Lett. B 525 (2002) 101 [hep-ph/0110146] [INSPIRE].

[35] M. Ibe, W. Nakano and M. Suzuki, Constraints on $L_{\mu}-L_{\tau}$ gauge interactions from rare kaon decay, Phys. Rev. D 95 (2017) 055022 [arXiv:1611.08460] [INSPIRE].

[36] A. Biswas, S. Choubey and S. Khan, Neutrino mass, dark matter and anomalous magnetic moment of muon in a $\mathrm{U}(1)_{L_{\mu}-L_{\tau}}$ model, JHEP 09 (2016) 147 [arXiv: 1608. 04194] [INSPIRE].

[37] F. Elahi and A. Martin, Constraints on $L_{\mu}-L_{\tau}$ interactions at the $L H C$ and beyond, Phys. Rev. D 93 (2016) 015022 [arXiv:1511.04107] [INSPIRE].

[38] S.N. Gninenko and N.V. Krasnikov, Probing the muon $g_{\mu}-2$ anomaly, $L_{\mu}-L_{\tau}$ gauge boson and dark matter in dark photon experiments, arXiv:1801.10448 [INSPIRE].

[39] J. Cao et al., Explaining the DAMPE data with scalar dark matter and gauged $\mathrm{U}(1)_{L_{e}-L_{\mu}}$ interaction, Eur. Phys. J. C 78 (2018) 198 [arXiv:1712.01244] [INSPIRE].

[40] A. Biswas, S. Choubey, L. Covi and S. Khan, Explaining the $3.5 \mathrm{keV} X$-ray line in a $L_{\mu}-L_{\tau}$ extension of the inert doublet model, JCAP 02 (2018) 002 [arXiv:1711.00553] [INSPIRE].

[41] S. Baek, Dark matter contribution to $b \rightarrow s \mu^{+} \mu^{-}$anomaly in local $\mathrm{U}(1)_{L_{\mu}-L_{\tau}}$ model, Phys. Lett. B 781 (2018) 376 [arXiv: 1707. 04573] [INSPIRE].

[42] F. Elahi and A. Martin, Using the modified matrix element method to constrain $L_{\mu}-L_{\tau}$ interactions, Phys. Rev. D 96 (2017) 015021 [arXiv:1705.02563] [INSPIRE].

[43] K. Asai, K. Hamaguchi and N. Nagata, Predictions for the neutrino parameters in the minimal gauged $\mathrm{U}(1)_{L_{\mu}-L_{\tau}}$ model, Eur. Phys. J. C 77 (2017) 763 [arXiv:1705.00419] [INSPIRE].

[44] Y. Kaneta and T. Shimomura, On the possibility of a search for the $L_{\mu}-L_{\tau}$ gauge boson at Belle-II and neutrino beam experiments, PTEP 2017 (2017) 053B04 [arXiv:1701.00156] [INSPIRE]. 
[45] A. Biswas, S. Choubey and S. Khan, FIMP and muon $(g-2)$ in a $\mathrm{U}(1)_{L_{\mu}-L_{\tau}}$ model, JHEP 02 (2017) 123 [arXiv: 1612.03067] [INSPIRE].

[46] Y. Tang and Y.-L. Wu, Flavor non-universal gauge interactions and anomalies in B-meson decays, Chin. Phys. C 42 (2018) 033104 [arXiv: 1705.05643] [INSPIRE].

[47] P. Ko, T. Nomura and H. Okada, A flavor dependent gauge symmetry, predictive radiative seesaw and LHCb anomalies, Phys. Lett. B 772 (2017) 547 [arXiv:1701.05788] [INSPIRE].

[48] G. Arcadi, T. Hugle and F.S. Queiroz, The dark $L_{\mu}-L_{\tau}$ rises via kinetic mixing, arXiv: 1803.05723 [INSPIRE].

[49] A. Kamada, K. Kaneta, K. Yanagi and H.-B. Yu, Self-interacting dark matter and muon $g-2$ in a gauged $\mathrm{U}(1)_{L_{\mu}-L_{\tau}}$ model, arXiv:1805.00651 [INSPIRE].

[50] A. Biswas, S. Choubey and S. Khan, Inverse seesaw and dark matter in a gauged B-L extension with flavour symmetry, arXiv:1805.00568 [INSPIRE].

[51] Z.-Z. Xing and Z.-H. Zhao, A review of $\mu-\tau$ flavor symmetry in neutrino physics, Rept. Prog. Phys. 79 (2016) 076201 [arXiv: 1512.04207] [INSPIRE].

[52] LHC Higgs Cross Section Working Group collaboration, D. de Florian et al., Handbook of LHC Higgs cross sections: 4. Deciphering the nature of the Higgs sector, arXiv: 1610.07922 [INSPIRE].

[53] LHC Higgs Cross Section Working Group collaboration, J.R. Andersen et al., Handbook of LHC Higgs cross sections: 3. Higgs properties, arXiv:1307.1347 [INSPIRE].

[54] CMS collaboration, Combined measurements of the Higgs boson's couplings at $\sqrt{s}=13 \mathrm{TeV}$, CMS-PAS-HIG-17-031, CERN, Geneva, Switzerland, (2017).

[55] ATLAS collaboration, Combined measurements of Higgs boson production and decay in the $H \rightarrow Z Z^{*} \rightarrow 4 \ell$ and $H \rightarrow \gamma \gamma$ channels using $\sqrt{s}=13$ TeV pp collision data collected with the ATLAS experiment, ATLAS-CONF-2017-047, CERN, Geneva, Switzerland, (2017).

[56] CMS collaboration, Measurements of Higgs boson properties in the diphoton decay channel in proton-proton collisions at $\sqrt{s}=13 \mathrm{TeV}$, arXiv: 1804.02716 [INSPIRE].

[57] ATLAS collaboration, Measurements of Higgs boson properties in the diphoton decay channel with $36 \mathrm{fb}^{-1}$ of pp collision data at $\sqrt{s}=13 \mathrm{TeV}$ with the ATLAS detector, arXiv: 1802.04146 [INSPIRE].

[58] ATLAS collaboration, Measurement of Higgs boson production in the diphoton decay channel in pp collisions at center-of-mass energies of 7 and $8 \mathrm{TeV}$ with the ATLAS detector, Phys. Rev. D 90 (2014) 112015 [arXiv:1408.7084] [INSPIRE].

[59] W. Altmannshofer and D.M. Straub, New physics in $B \rightarrow K^{*} \mu \mu$ ?, Eur. Phys. J. C 73 (2013) 2646 [arXiv: 1308.1501] [inSPIRE].

[60] W. Altmannshofer and D.M. Straub, New physics in $b \rightarrow s$ transitions after LHC run 1, Eur. Phys. J. C 75 (2015) 382 [arXiv:1411.3161] [InSPIRE].

[61] W. Altmannshofer and D.M. Straub, Implications of $b \rightarrow s$ measurements, in Proceedings, $50^{\text {th }}$ Rencontres de Moriond Electroweak Interactions and Unified Theories, La Thuile, Italy, 14-21 March 2015, pg. 333 [arXiv: 1503.06199] [INSPIRE].

[62] A. Greljo, G. Isidori and D. Marzocca, On the breaking of lepton flavor universality in $B$ decays, JHEP 07 (2015) 142 [arXiv: 1506.01705] [INSPIRE]. 
[63] S. Descotes-Genon, L. Hofer, J. Matias and J. Virto, Global analysis of $b \rightarrow$ sll anomalies, JHEP 06 (2016) 092 [arXiv: 1510.04239] [INSPIRE].

[64] T. Hurth, F. Mahmoudi and S. Neshatpour, On the anomalies in the latest LHCb data, Nucl. Phys. B 909 (2016) 737 [arXiv:1603.00865] [InSPIRE].

[65] W. Altmannshofer, P. Stangl and D.M. Straub, Interpreting hints for lepton flavor universality violation, Phys. Rev. D 96 (2017) 055008 [arXiv: 1704.05435] [INSPIRE].

[66] B. Capdevila, A. Crivellin, S. Descotes-Genon, J. Matias and J. Virto, Patterns of new physics in $b \rightarrow s \ell^{+} \ell^{-}$transitions in the light of recent data, JHEP 01 (2018) 093 [arXiv: 1704.05340] [INSPIRE].

[67] W. Altmannshofer, C. Niehoff, P. Stangl and D.M. Straub, Status of the $B \rightarrow K^{*} \mu^{+} \mu^{-}$ anomaly after Moriond 2017, Eur. Phys. J. C 77 (2017) 377 [arXiv:1703.09189] [InSPIRE].

[68] BeLLE collaboration, S. Wehle et al., Lepton-flavor-dependent angular analysis of $B \rightarrow K^{*} \ell^{+} \ell^{-}$, Phys. Rev. Lett. 118 (2017) 111801 [arXiv:1612.05014] [InSPIRE].

[69] CMS collaboration, Search for narrow resonances in dilepton mass spectra in proton-proton collisions at $\sqrt{s}=13 \mathrm{TeV}$ and combination with $8 \mathrm{TeV}$ data, Phys. Lett. B 768 (2017) 57 [arXiv: 1609.05391] [INSPIRE].

[70] ATLAS collaboration, Search for high-mass new phenomena in the dilepton final state using proton-proton collisions at $\sqrt{s}=13 \mathrm{TeV}$ with the ATLAS detector, Phys. Lett. B 761 (2016) 372 [arXiv: 1607.03669] [INSPIRE].

[71] ATLAS collaboration, Search for new high-mass phenomena in the dilepton final state using $36 \mathrm{fb}^{-1}$ of proton-proton collision data at $\sqrt{s}=13 \mathrm{TeV}$ with the ATLAS detector, JHEP 10 (2017) 182 [arXiv: 1707.02424] [INSPIRE].

[72] ATLAS collaboration, Search for high-mass dilepton resonances in pp collisions at $\sqrt{s}=8 \mathrm{TeV}$ with the ATLAS detector, Phys. Rev. D 90 (2014) 052005 [arXiv:1405.4123] [INSPIRE].

[73] ATLAS collaboration, Search for high-mass resonances decaying to dilepton final states in pp collisions at $\sqrt{s}=7 \mathrm{TeV}$ with the ATLAS detector, JHEP 11 (2012) 138 [arXiv: 1209.2535] [INSPIRE].

[74] CMS collaboration, Searches for dijet resonances in pp collisions at $\sqrt{s}=13 \mathrm{TeV}$ using data collected in 2016, CMS-PAS-EXO-16-056, CERN, Geneva, Switzerland, (2016).

[75] W. Altmannshofer, S. Gori, M. Pospelov and I. Yavin, Neutrino trident production: a powerful probe of new physics with neutrino beams, Phys. Rev. Lett. 113 (2014) 091801 [arXiv:1406.2332] [INSPIRE].

[76] CHARM-II collaboration, D. Geiregat et al., First observation of neutrino trident production, Phys. Lett. B 245 (1990) 271 [INSPIRE].

[77] CCFR collaboration, S.R. Mishra et al., Neutrino tridents and WZ interference, Phys. Rev. Lett. 66 (1991) 3117 [INSPIRE].

[78] NuTeV collaboration, T. Adams et al., Neutrino trident production from $\mathrm{NuTeV}$, in High-energy physics. Proceedings, $29^{\text {th }}$ International Conference, ICHEP'98, Vancouver, Canada, 23-29 July 1998, pg. 631 [hep-ex/9811012] [INSPIRE].

[79] K. Fuyuto, W.-S. Hou and M. Kohda, $Z^{\prime}$-induced FCNC decays of top, beauty and strange quarks, Phys. Rev. D 93 (2016) 054021 [arXiv: 1512.09026] [INSPIRE]. 
[80] D. Liu, J. Liu, C.E.M. Wagner and X.-P. Wang, Bottom-quark forward-backward asymmetry, dark matter and the LHC, Phys. Rev. D 97 (2018) 055021 [arXiv:1712.05802] [INSPIRE].

[81] ATLAS collaboration, Search for R-parity-violating supersymmetric particles in multi-jet final states produced in pp collisions at $\sqrt{s}=13 \mathrm{TeV}$ using the ATLAS detector at the LHC, arXiv: 1804.03568 [INSPIRE].

[82] ATLAS collaboration, Search for massive supersymmetric particles decaying to many jets using the ATLAS detector in pp collisions at $\sqrt{s}=8$ TeV, Phys. Rev. D 91 (2015) 112016 [Erratum ibid. D 93 (2016) 039901] [arXiv: 1502.05686] [INSPIRE].

[83] CMS collaboration, Searches for light- and heavy-flavour three-jet resonances in pp collisions at $\sqrt{s}=8 \mathrm{TeV}$, Phys. Lett. B 730 (2014) 193 [arXiv:1311.1799] [INSPIRE].

[84] S. El Hedri, A. Kaminska, M. de Vries and J. Zurita, Simplified phenomenology for colored dark sectors, JHEP 04 (2017) 118 [arXiv:1703.00452] [INSPIRE].

[85] M. Czakon, M.L. Mangano, A. Mitov and J. Rojo, Constraints on the gluon PDF from top quark pair production at hadron colliders, JHEP 07 (2013) 167 [arXiv:1303.7215] [INSPIRE].

[86] R. Barbieri and G.F. Giudice, $b \rightarrow s \gamma$ decay and supersymmetry, Phys. Lett. B 309 (1993) 86 [hep-ph/9303270] [INSPIRE].

[87] Particle Data Group collaboration, C. Patrignani et al., Review of particle physics, Chin. Phys. C 40 (2016) 100001 [inSPIRE].

[88] A.J. Buras, F. De Fazio and J. Girrbach, The anatomy of $Z^{\prime}$ and $Z$ with flavour changing neutral currents in the flavour precision era, JHEP 02 (2013) 116 [arXiv:1211.1896] [INSPIRE].

[89] G. Isidori, Flavor physics and CP-violation, in Proceedings, 2012 European School of High-Energy Physics (ESHEP 2012), La Pommeraye, Anjou, France, 6-19 June 2012, CERN-2014-008, (2014), pg. 69 [arXiv:1302.0661] [INSPIRE].

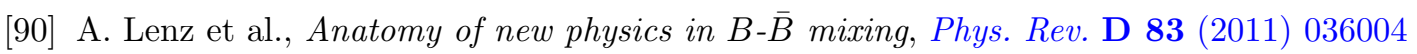
[arXiv: 1008.1593] [INSPIRE].

[91] ATLAS collaboration, A. Durglishvili, Search for $t Z$ flavour changing neutral currents in top-quark decays with the ATLAS detector, arXiv:1712.09802 [INSPIRE]. 